\title{
Canonical coordinates for the planetary problem*
}

\author{
Gabriella Pinzari \\ Dipartimento di Matematica ed Applicazioni "R. Caccioppoli" \\ Università di Napoli "Federico II" \\ Monte Sant'Angelo - Via Cinthia I-80126 Napoli (Italy) \\ gabriella.pinzari@unina.it
}

November, 3, 2014

\begin{abstract}
We collect classical and more recent material about possible symplectic descriptions of the phase space of the planetary problem.
\end{abstract}

Keywords: Canonical coordinates. Planetary problem. Delaunay-Poincaré coordinates. Jacobi's reduction of the nodes. Deprit's reduction of the nodes. RPS variables. Keplerian ellipses. Symmetries. Perihelia reduction.

MSC2000 numbers: 34-01, 37-01, 70-01, 34-02, 37-02, 70-02

\section{Contents}

1 Introduction 2

2 Linear momentum reductions 5

3 Canonical coordinates fitted to the perturbative setting $\quad 7$

3.1 On the canonical integration of the two-body problem (Delaunay and Poincaré

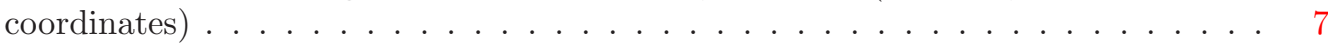

3.2 Kepler maps . . . . . . . . . . . . . . . . . . . . . . . . . . . . . . 10

3.3 Dynamical equivalence of the heliocentric and Jacobi reduction . . . . . . . . . 12

4 Examples of canonical Kepler maps 13

4.1 Jacobi-Radau's reduction of the nodes for three bodies . . . . . . . . . . . . . . . 14

4.2 Boigey-Deprit's reduction for $(1+n)$ bodies . . . . . . . . . . . . 16

4.3 Planetary version of Deprit's variables and regularizations . . . . . . . . . . . 20

4.3.1 Partial reduction (RPS variables) . . . . . . . . . . . . . . 21

4.3 .2 Full reduction . . . . . . . . . . . . . . . . . . . . . 22

4.4 Full reduction and reflection symmetries . . . . . . . . . . . . . . . 22

4.5 "Perihelia reduction": A symmetric full reduction of $\mathrm{SO}(3)$ invariance . . . . . . . 23

*Research supported by ERC Ideas-Project 306414 "Hamiltonian PDEs and small divisor problems: a dynamical systems approach" and STAR Project of Federico II University, Naples. 


\section{Introduction}

1.1 In 1963, V. I. Arnold stated his celebrated Theorem ${ }^{1}$ on the Stability of Planetary Motions (hereafter, Arnold's Theorem). The general proof of his wonderful statement (that he provided completely only for the particular case of three bodies constrained on a plane) turned out to be more difficult than expected and was next completed by J. Laskar, P. Robutel, M. Herman, J. Féjoz, L. Chierchia and the author. We refer the reader to the technical papers [2], [19], [27], [21], [16], [11], [23], [8] for detailed information; to [12], [4], [9], or the introduction of [24] for reviews.

The complete understanding of Arnold's Theorem relied on an analytic part and a geometric one, both highly non trivial. Of such two aspects, the analytic part was basically settled out since [2] (notwithstanding refinements next given in [11], [5]). The geometrical aspects, of which Arnold just provided, for the general case, only sketchy conjectures, were instead mostly unexplored after his 1963's paper and have been only recently clarified [23], [8], [25].

In fact, switching from the three-body case to the many-body one needed to develop new constructions not known at those times.

The purpose of this note is to provide a historical survey of this latter part. We shall describe previous classical approaches going back to Delaunay, Poincaré, Jacobi and point out more recent progresses, based on the papers [23], [6], [8], [7], [24], [25].

We begin with introducing the problem.

The planetary problem consists in studying the motion of $(1+n)$ point masses, a "sun" and $n$ "planets" interacting through gravity. This is a Hamiltonian problem: If the masses of the sun and of the planets are denoted, respectively, as $m_{0}, \mu m_{1}, \cdots, \mu m_{n}$, where $\mu$ is a very small number, and the Euclidean length as $|\cdot|$, the motion equations may be written in the form of Hamilton equation where the Hamiltonian (the energy ${ }^{2}$ ) of the system is

$$
\mathrm{H}_{(1+n) \mathrm{b}}(p, q)=\sum_{1 \leq i \leq n} \frac{\left|p^{(i)}\right|^{2}}{2 m_{i}}-\sum_{1 \leq i \leq n} \frac{m_{0} m_{i}}{\left|q^{(0)}-q^{(i)}\right|}+\mu \frac{\left|p^{(0)}\right|^{2}}{2 m_{0}}-\mu \sum_{1 \leq i<j \leq n} \frac{m_{i} m_{j}}{\left|q^{(i)}-q^{(j)}\right|} .
$$

Here, for $0 \leq i \leq n, 1 \leq j \leq 3, q=\left(q^{(0)}, \cdots, q^{(n)}\right)$, with $q^{(i)}=\left(q_{1}^{(i)}, q_{2}^{(i)}, q_{3}^{(i)}\right)$ are the planets' positions and $p=\left(p^{(0)}, \cdots, p^{(n)}\right)$, with $p^{(i)}=\left(p_{1}^{(i)}, p_{2}^{(i)}, p_{3}^{(i)}\right)$, their conjugated momenta.

Despite of its apparent $(3+3 n)$ degrees of freedom $((2 n+2)$ for the problem in the plane), $\mathrm{H}_{(1+n) \mathrm{b}}$ possesses a variety of integrals. These are: the three components of the center of mass $\mathrm{Q}=\left(m_{0} q^{(0)}+\mu \sum_{1 \leq i \leq n} m_{i} q^{(i)}\right) /$ const; the three components of the total linear momentum $\mathrm{P}=$ $\sum_{0 \leq i \leq n} p^{(i)}=$ const $\mathrm{Q}$; the three components of the total angular momentum $\mathrm{C}=\sum_{0 \leq i \leq n} q^{(i)} \times$ $p^{(i)}$ and the energy $\mathrm{E}=\mathrm{H}_{(1+n) \mathrm{b}}$. Here, "const" is the sum of the masses and " $\times$ " denotes skew-product.

Poincaré proved that no other analytic integral for the system may be found, apart for the ten ones listed above. They are more than enough to integrate the problem "by quadratures" in the case of two bodies $(n=1$; see $\S 3.1)$. When $n \geq 2$, the problem is non-integrable. Its dynamics may be very complicated and exhibit also chaotic behaviors [13].

The integrals above are independent but do not commute. It is however possible to isolate among them five independent and pairwise commuting quantities, for the problem in the space; three of them for the problem constrained on a plane. These are: the three components of the linear

\footnotetext{
${ }^{1}$ [2, Chapter III, p. 125] "For the majority of initial conditions under which the instantaneous orbits of the planets are close to circles lying in a single plane, perturbation of the planets on one another produces, in the course of an infinite interval of time, little change on these orbits provided the masses of the planets are sufficiently small. [...] In particular [...] in the n-body problem there exists a set of initial conditions having a positive Lebesgue measure and such that, if the initial positions and velocities of the bodies belong to this set, the distances of the bodies from each other will remain perpetually bounded."

${ }^{2}$ More precisely, $\mu \mathrm{H}_{(1+n) \mathrm{b}}(p / \mu, q)$ corresponds to be the mechanical energy $T+U$.
} 
momentum $\mathrm{P}$, the third component $\mathrm{C}_{3}$ of the total angular momentum $\mathrm{C}$ and ${ }^{3}$ its Euclidean length $\mathrm{G}:=|\mathrm{C}|$ in the former case; the two components of $\mathrm{P}$ and $\mathrm{C}_{3}$, in the latter. This tells us that the "effective" number of degrees of freedom is $(3 n+3)-5=3 n-2$ in the space; $(2 n+2)-3=2 n-1$ in the plane.

To any of such independent integrals one associates (by Noether theorem) a one-parameter group of symplectic transformations which leave $\mathrm{H}_{(1+n) \mathrm{b}}$ unvaried. For problem in the space, these are

- translations

$$
\mathrm{t}_{a}:\left(p^{(i)}, q^{(i)}\right) \rightarrow\left(p^{(i)}, q^{(i)}+a\right) \quad a \in \mathbb{R}^{3}
$$

with respect to three independent directions $a=\left(a_{1}, a_{2}, a_{3}\right)$;

- "synchronous" rotations for the $p$ and the $q$-variables about the $k^{(3)}$-axis:

$$
\mathrm{R}_{\alpha}^{(3)}:\left(p^{(i)}, q^{(i)}\right) \rightarrow\left(\mathrm{R}_{\alpha}^{(3)} p^{(i)}, \mathrm{R}_{\alpha}^{(3)} q^{(i)}\right) \quad \alpha \in \mathbb{T} ;
$$

- or about the C-axis:

$$
\mathrm{R}_{\beta}^{\mathrm{C}}:\left(p^{(i)}, q^{(i)}\right) \rightarrow\left(\mathrm{R}_{\beta}^{\mathrm{C}} p^{(i)}, \mathrm{R}_{\beta}^{\mathrm{C}} q^{(i)}\right) \quad \beta \in \mathbb{T} .
$$

Here, $\mathrm{F}_{0}=\left(k^{(1)}, k^{(2)}, k^{(3)}\right)$ is a prefixed reference frame, $\mathbb{T}:=\mathbb{R} / 2 \pi \mathbb{Z}$ is the flat torus and $\mathrm{R}_{\alpha}^{(3)}, \mathrm{R}_{\beta}^{\mathrm{C}}$ are suitable $3 \times 3$ matrices having the form of $\mathrm{R}^{(3)}(\mathrm{h})$ below (see Eq. (13)) in suitable reference frames, with $\mathrm{h}=\alpha, \beta$.

For the problem in the plane one has analogous symmetries.

We remark that, since the transformations listed above are caused by integrals, unless such integrals are eliminated in some way, they are present in the Hamiltonian (in different forms), no matter what system of coordinates is used. Arnold in 1963 argued that the symmetry by rotations plays a key rôle in order to overcome the problem of the "proper degeneracy" (that we shall recall in §3.1) of the planetary system, since it implies the existence of an elliptic equilibrium point for the secular ${ }^{4}$ system written in Poincaré coordinates (see §3.1) in correspondence of circular, planar motions. He foresaw this equilibrium point might be a bifurcation point of full-dimensional invariant tori surrounded of quasi-periodic motions. Quite paradoxically, the invariance by rotations itself is just the origin of a strong degeneracy, for the problem in the space, that, if not suitably treated, strongly prevents the direct application of his project. Arnold's program was then successfully completed in [27] and, in its full generality, in [23], [8].

It is worth to recall also that further symmetries for $\mathrm{H}_{(1+n) \mathrm{b}}$ do exist for the planetary Hamiltonian which are not related to integrals.

These are

- "asynchronous" rotations for the $p$ and the $q$-variables, i.e.,

$$
\left(p^{(i)}, q^{(i)}\right) \rightarrow\left(\mathrm{R} p^{(i)}, \mathrm{S} q^{(i)}\right)
$$

where $\mathrm{RR}^{\mathrm{t}}=\mathrm{id}=\mathrm{SS}^{\mathrm{t}}$, with ${ }^{\mathrm{t}}$ denoting transpose and $\mathrm{R} \neq \mathrm{S}$;

- "reflections", i.e., transformations of the form

$$
\left(\left(y_{1}^{(i)}, y_{2}^{(i)}, y_{3}^{(i)}\right),\left(x_{1}^{(i)}, x_{2}^{(i)}, x_{3}^{(i)}\right)\right) \rightarrow\left(\left(r_{1} y_{1}^{(i)}, r_{2} y_{2}^{(i)}, r_{3} y_{3}^{(i)}\right),\left(s_{1} x_{1}^{(i)}, s_{2} x_{2}^{(i)}, s_{3} x_{3}^{(i)}\right)\right)
$$

with $r_{j}, s_{j}= \pm 1$.

These symmetries play an important rôle in the case one would like to know whether it is possible to reduce completely the number of degrees of freedom of the system and, simultaneously, keep some parity (compare also the next paragraph).

\footnotetext{
${ }^{3}$ Recall that the three components of $\mathrm{C}$ do not commute. Rather, they verify $\left\{\mathrm{C}_{i}, \mathrm{C}_{j}\right\}=\delta_{i j k} \mathrm{C}_{k}$, where $\delta_{i j k}$ is the Levi-Civita symbol.

${ }^{4}$ In this context, "secular system" is used to denote a suitable time-average of it. A rigorous definition will be given in $\S 3.3$ below.
} 
1.2 In general for a quasi-integrable system

$$
\mathrm{H}(I, \varphi)=h(I)+\varepsilon f(I, \varphi) \quad(I, \varphi) \in V \times \mathbb{T}^{n} \quad V \subset \mathbb{R}^{n} \quad \text { open }
$$

"natural" action-angle coordinates $(I, \varphi)$ are uniquely determined (up to isomorphisms) by the integrable part $h$.

In the case of the planetary problem, notwithstanding its clear quasi-integrable structure (it is close to the integrable problem consisting of the uncoupled interaction of each planet with the sun), its proper degeneracies mentioned above imply that the choice of symplectic coordinates is not uniquely determined. We then aim to describe possible sets of canonical coordinates which let the system free of its integrals (all of, or just a part of them). We shall only deal with systems of coordinates such that the unperturbed part takes the aspect of the classical Keplerian form (see Eq. (16) below). See also [28] and references therein for further examples.

In order to reduced symmetries due to integrals, one has to deal with the linear momentum $\mathrm{P}$ and the angular momentum $\mathrm{C}$.

The reduction of the linear momentum may be performed in two (equivalent) ways, as described in $\S 2$, switching to Jacobi or heliocentric coordinates.

The reduction of the angular momentum does not present difficulties in the case of the planar problem and can be performed using common tools of canonical transformations. Therefore, we shall not deal about it (see however [7] for recent results concerning the Birkhoff normal form for the completely reduced problem in the planar case).

The reduction of the angular momentum for the problem in the space is instead something more intriguing.

For a long time, only the spatial three-body case could be handled, by means of a tool, known since the XIX century, under the name of "reduction of the nodes", developed by Jacobi and Radau [17], [26]. It has been used in [27] to prove Arnold's Theorem in this case and is recalled in $\S 4.1$.

In lack of coordinates fitted to reduce completely rotations for the spatial problem in the case of more than three bodies, many authors [1], [16], [11], [22] used classical sets coordinates named after Delaunay and Poincaré. Delaunay and Poincaré coordinates (which are recalled in §3.1) are suited to the quasi-integrability of the problem, but not to its symmetry by rotations.

Only recently, new systems of coordinates have been applied to the problem [23], [8], in order to prove directly Arnold's Theorem and estimating the measure of its stable motions, in terms of the maximum of eccentricities and inclinations of unperturbed Keplerian motions. These coordinates are related to a certain set discovered in the 80 's by A. Deprit [10], and re-discovered by the author during her $\mathrm{PhD}$ [23], which reduce completely the number of degrees of freedom of the system. Deprit's coordinates generalize to an arbitrary number of planets the reduction of the nodes by Jacobi and Radau, and, as well as it, are defined only for the problem in the space. To overcome the problem of singularity of the coordinates for planar motions, in [23], [8] (see also a conjecture in [2] and a formal construction in [21]), starting from Deprit's coordinates, a system of coordinates realizing just a partial reduction has been introduced. This allows to let the system free from its rotational degeneracy mentioned above (and hence to obtain the complete proof of Arnold's theorem), even though with an extra degree of freedom. With this extra-degree of freedom it is also possible to treat the spatial and the planar problem in a unified way: the partially reduced coordinates of the spatial case are defined also in the planar case, where they reduce to the Poincaré (hence, unreduced) planar coordinates. This part of the story is told in the following $\S 4.2-\S 4.3$.

We now remark that, as it follows from the papers [8] and [7], complete, global reductions of the number of degrees of freedom are available both for the planar and the spatial problem separately. This has the following physical consequence: almost co-planar quasi-periodic motions of the spatial problem with the minimum number of degrees of freedom need not to be closer and closer to the corresponding motions of the planar problem. Clearly, one should reasonably expect 
the opposite situation. It is to add that a certain continuity between the spatial and the planar three body problem was invoked by Arnold since [2] and this turned out to be a controversial question widely discussed since M. Herman's investigations to this problem. In $\S 4.4-\S 4.5$ we discuss the problem of having a unique, well defined, global system of coordinates for the spatial and the planar problem. We shall present a system of coordinates (which we name "perihelia reduction") which does this job and, moreover, keeps parities due to reflections. This new set reveals to be useful to prove (besides the aforementioned continuity) that quasi-periodic motions with minimum number of independent frequencies do exist even away from Arnold's elliptic equilibrium, i.e., for relatively large eccentricities and inclinations. See [25] for an announcement of this result.

\section{Linear momentum reductions}

The first step consists into eliminating the motion of the sun by fixing the coordinates of the center of mass Q. This leads to a $3 n$-degrees of freedom Hamiltonian which governs the motion of the planets. The methods used in literature to achieve this reduction are essentially two, respectively referred to as the heliocentric reduction and the reduction via Jacobi coordinates.

The heliocentric reduction has been used in [16], [11], [27], [8] and goes as follows. One performs the change of variables

$$
\left\{\begin{aligned}
x^{(0)} & :=q^{(0)} \\
x^{(i)} & :=q^{(i)}-q^{(0)} \quad 1 \leq i \leq n
\end{aligned}\right.
$$

$$
\left\{\begin{array}{l}
y^{(0)}:=\sum_{i=0}^{n} p^{(i)}=\mathrm{P} \\
y^{(i)}:=p^{(i)} \quad 1 \leq i \leq n .
\end{array}\right.
$$

The change is linear, canonical (more precisely, it is homogeneous ${ }^{5}$ ). The conservation of $y^{(0)}=\mathrm{P}$ implies $x^{(0)}$ is cyclic and, on the manifold where $\mathrm{Q}$ is constant, we have $\mathrm{P}=0$. Therefore, we can conveniently fix $x^{(0)}=0$ and $\mathrm{P}=0$ in order to find the expression of the reduced Hamiltonian. This amounts to take

$$
\left\{\begin{array} { l } 
{ q ^ { ( 0 ) } = 0 } \\
{ q ^ { ( i ) } = x ^ { ( i ) } \quad 1 \leq i \leq n }
\end{array} \quad \left\{\begin{array}{l}
p^{(0)}=-\sum_{i=1}^{n} y^{(i)} \\
p^{(i)}=y^{(i)} \quad 1 \leq i \leq n .
\end{array}\right.\right.
$$

Correspondingly, we find for the "heliocentric Hamiltonian" the expression

$$
\begin{aligned}
\mathrm{H}_{\text {hel }}(y, x) & =\sum_{i=1}^{n}\left(\frac{\left|y^{(i)}\right|^{2}}{2 \mathfrak{m}_{i}}-\frac{\mathfrak{m}_{i} \mathfrak{M}_{i}}{\left|x^{(i)}\right|}\right)+\mu \sum_{1 \leq i<j \leq n}\left(\frac{y^{(i)} \cdot y^{(j)}}{m_{0}}-\frac{m_{i} m_{j}}{\left|x^{(i)}-x^{(j)}\right|}\right) \\
& =: \sum_{i=1}^{n}\left(\frac{\left|y^{(i)}\right|^{2}}{2 \mathfrak{m}_{i}}-\frac{\mathfrak{m}_{i} \mathfrak{M}_{i}}{\left|x^{(i)}\right|}\right)+\mu f_{\text {hel }}(y, x)
\end{aligned}
$$

where $\mathfrak{m}_{i}:=\frac{m_{0} m_{i}}{m_{0}+\mu m_{i}}$ and $\mathfrak{M}_{i}:=m_{0}+\mu m_{i}$.

The reduction via Jacobi coordinates has been used, for example, in [2], [29]. It works well when the number of planets is small. For simplicity, we present it for the three-body case; generalizations may be obtained.

\footnotetext{
${ }^{5}$ As usual, by "canonical, we mean that the standard 2-form $\sum_{0 \leq i \leq n} \sum_{1 \leq j \leq 3} d p_{j}^{(i)} \wedge d q_{j}^{(i)}$ is preserved. By "homogeneous", we mean that the standard 1-form $\sum_{0 \leq i \leq n} \sum_{1 \leq j \leq 3} p_{j}^{(i)} d q_{j}^{(i)}$ is preserved.
} 
Consider three masses, $m_{0}, \mu m_{1}$ and $\mu m_{2}$. Let

$$
\mathrm{Q}^{\prime}:=\frac{m_{0} q^{(0)}+\mu m_{1} q^{(1)}}{m_{0}+\mu m_{1}}
$$

the center of mass of $m_{0}$ and $\mu m_{1}$ and switch to the (homogeneous-canonical) coordinates

$$
\left\{\begin{array} { l } 
{ \tilde { x } ^ { ( 0 ) } = q ^ { ( 0 ) } } \\
{ \tilde { x } ^ { ( 1 ) } = q ^ { ( 1 ) } - q ^ { ( 0 ) } } \\
{ \tilde { x } ^ { ( 2 ) } = q ^ { ( 2 ) } - \mathrm { Q } ^ { \prime } }
\end{array} \quad \left\{\begin{array}{l}
\tilde{y}^{(0)}=p^{(0)}+p^{(1)}+p^{(2)} \\
\tilde{y}^{(1)}=p^{(1)}+\frac{\mu m_{1}}{m_{0}+\mu m_{1}} p^{(2)} \\
\tilde{y}^{(2)}=p^{(2)} .
\end{array}\right.\right.
$$

Proceeding in a similar way as for the heliocentric reduction (i.e., using the cyclic character of $\widetilde{x}^{(0)}$ and restricting to the manifold where $\mathrm{Q}$ is constant, which allows to take also $\tilde{y}^{(0)}=0$ ) the expression of the transformed Hamiltonian can be found taking

$$
\left\{\begin{array} { l } 
{ q ^ { ( 0 ) } = 0 } \\
{ q ^ { ( 1 ) } = \tilde { x } ^ { ( 1 ) } } \\
{ q ^ { ( 2 ) } = \frac { \mu m _ { 1 } } { m _ { 0 } + \mu m _ { 1 } } \tilde { x } ^ { ( 1 ) } + \tilde { x } ^ { ( 2 ) } }
\end{array} \quad \left\{\begin{array}{l}
p^{(0)}=-\tilde{y}^{(1)}-\frac{m_{0}}{m_{0}+\mu m_{1}} \tilde{y}^{(2)} \\
p^{(1)}=\tilde{y}^{(1)}-\frac{\mu m_{1}}{m_{0}+\mu m_{1}} \tilde{y}^{(2)} \\
p^{(2)}=\tilde{y}^{(2)} .
\end{array}\right.\right.
$$

Correspondingly, one finds the "Jacobi-Hamiltonian"

$$
\begin{aligned}
\mathrm{H}_{\mathrm{Jac}} & =\frac{\left|\tilde{y}^{(1)}\right|^{2}}{2 \tilde{\mathfrak{m}}_{1}}+\frac{\left|\tilde{y}^{(2)}\right|^{2}}{2 \tilde{\mathfrak{m}}_{2}}-\frac{m_{0} m_{1}}{\left|\tilde{x}^{(1)}\right|}-\frac{m_{0} m_{2}}{\left|\tilde{x}^{(2)}+\frac{\mu m_{1}}{m_{0}+\mu m_{1}} \tilde{x}^{(1)}\right|} \\
& -\mu \frac{m_{1} m_{2}}{\left|\frac{m_{0}}{m_{0}+\mu m_{1}} \tilde{x}^{(1)}-\tilde{x}^{(2)}\right|}
\end{aligned}
$$

that we write in the form

$$
\mathrm{H}_{\mathrm{Jac}}=: \quad \sum_{i=1}^{2}\left(\frac{\left|\tilde{y}^{(i)}\right|^{2}}{2 \tilde{\mathfrak{m}}_{i}}-\frac{\tilde{\mathfrak{m}}_{i} \tilde{\mathfrak{M}}_{i}}{\left|x^{(i)}\right|}\right)+\mu f_{\mathrm{Jac}}(\tilde{y}, \tilde{x})
$$

with $\tilde{\mathfrak{m}}_{1}=\mathfrak{m}_{1}=\frac{m_{0} m_{1}}{m_{0}+\mu m_{1}}, \tilde{\mathfrak{m}}_{2}=\frac{m_{2}\left(m_{0}+\mu m_{1}\right)}{m_{0}+\mu m_{1}+\mu m_{2}}=\mathfrak{m}_{2}+\mathrm{O}\left(\mu^{2}\right), \tilde{\mathfrak{M}}_{1}:=\mathfrak{M}_{1}=m_{0}+\mu m_{1}$, $\tilde{\mathfrak{M}}_{2}:=m_{0} \frac{m_{0}+\mu m_{1}+\mu m_{2}}{m_{0}+\mu m_{1}}$.

The choice of $\tilde{\mathfrak{M}}_{i}$ is justified by the fact that, if one takes the expansion of the two last terms of $\mathrm{H}_{\mathrm{Jac}}$ in powers of $\mu$, then he finds the Hamiltonian

$$
\widetilde{\mathrm{H}}_{\mathrm{Jac}}=\sum_{1 \leq i \leq 2}\left(\frac{\left|\tilde{y}^{(i)}\right|^{2}}{2 \tilde{\mathfrak{m}}_{i}}-\frac{\tilde{\mathfrak{m}}_{i} \tilde{\mathfrak{M}}_{i}}{\left|\tilde{x}^{(i)}\right|}\right)+\mu m_{1} m_{2}\left(\frac{\tilde{x}^{(1)} \cdot \tilde{x}^{(2)}}{\left|\tilde{x}^{(2)}\right|^{3}}-\frac{1}{\left|\tilde{x}^{(1)}-\tilde{x}^{(2)}\right|}\right),
$$

which differs from $\mathrm{H}_{\mathrm{Jac}}$ by $\mathrm{O}\left(\mu^{2}\right)$. The Hamiltonian $\widetilde{\mathrm{H}}_{\mathrm{Jac}}$ has been proposed in [2] for the proof of Arnold's theorem.

The relation between the heliocentric and the Jacobi reduction is simple. 
The definitions above imply that, if $\left(y^{(1)}, y^{(2)}, x^{(1)}, x^{(2)}\right)=\phi_{\text {hel } / \mathrm{Jac}}\left(\tilde{y}^{(1)}, \tilde{y}^{(2)}, \tilde{x}^{(1)}, \tilde{x}^{(2)}\right)$ denotes the linear, symplectic, close to the identity transformation given by

$$
\left\{\begin{array} { l } 
{ x ^ { ( 1 ) } = \tilde { x } ^ { ( 1 ) } } \\
{ x ^ { ( 2 ) } = \frac { \mu m _ { 1 } } { m _ { 0 } + \mu m _ { 1 } } \tilde { x } ^ { ( 1 ) } + \tilde { x } ^ { ( 2 ) } }
\end{array} \quad \left\{\begin{array}{l}
y^{(1)}=\tilde{y}^{(1)}-\frac{\mu m_{1}}{m_{0}+\mu m_{1}} \tilde{y}^{(2)} \\
y^{(2)}=\tilde{y}^{(2)}
\end{array}\right.\right.
$$

then the heliocentric and the Jacobi Hamiltonian are related by

$$
\mathrm{H}_{\mathrm{Jac}}=\mathrm{H}_{\mathrm{hel}} \circ \phi_{\mathrm{hel} / \mathrm{Jac}} .
$$

From this relation, a certain "equivalence" (in the sense of normal form) between $\mathrm{H}_{\mathrm{Jac}}, \mathrm{H}_{\text {hel }}$ follows, that will be discussed later (compare $§ 3.3$ ).

\section{Canonical coordinates fitted to the perturbative setting}

Whatever is the method which one chooses to eliminate the coordinates of the sun (heliocentric, Jacobi or others), the resulting Hamiltonian for the planets takes the form of a quasi-integrable system, where

- the leading part (of "order one") is a sum of uncoupled "two-body systems" of the form

$$
h_{2 \mathrm{~b}}^{(i)}=\frac{\left|y^{(i)}\right|^{2}}{2 \mathfrak{m}_{i}}-\frac{\mathfrak{m}_{i} \mathfrak{M}_{i}}{\left|x^{(i)}\right|},
$$

which, as well known, are integrable;

- the perturbing function (of "order $\mu$ "), as well as $h_{2 \mathrm{~b}}^{(i)}$, possesses the integral

$$
\mathrm{C}=\sum_{1 \leq i \leq n} \mathrm{C}^{(i)}
$$

where $\mathrm{C}^{(i)}=x^{(i)} \times y^{(i)}$.

\subsection{On the canonical integration of the two-body problem (Delaunay and Poincaré coordinates)}

Referring to classical textbooks for more details, in this section we recall a few facts about the construction of canonical variables for $h_{2 \mathrm{~b}}^{(i)}$.

One often says that $h_{2 \mathrm{~b}}^{(i)}$ is "super-integrable". This locution reflects the fact that $h_{2 \mathrm{~b}}^{(i)}$, despite of having three degrees of freedom, possesses five independent integrals, even more than its degrees of freedom. These are: the three components of the angular momentum $\mathrm{C}^{(i)}=x^{(i)} \times y^{(i)}$, the direction of the so-called eccentricity vector $\mathrm{L}^{(i)}$, which is perpendicular to $\mathrm{C}^{(i)}$ and the energy. The presence of so many integrals causes two degeneracies in the integration of $h_{2 \mathrm{~b}}^{(i)}$, which are often referred to as "proper degeneracies". More precisely, degeneracies arise from the fact that, excluding the energy, the four remaining integrals may be rearranged into two couples of canonical conjugated variables $\left(\left(\mathrm{H}_{i}, \mathrm{~h}_{i}\right)\right.$ and $\left(\Gamma_{i}, \mathrm{~g}_{i}\right)$ below $)$. Let us recall this construction.

The integrals above are not in all involution. Then one chooses the Euclidean length $\left|\mathrm{C}^{(i)}\right|$ and the third component $\mathrm{C}_{3}^{(i)}=\mathrm{C}^{(i)} \cdot k^{(3)}$ (where $\mathrm{F}_{0}=\left(k^{(1)}, k^{(2)}, k^{(3)}\right)$ is a prefixed orthonormal triple in $\mathbb{R}^{3}$ ), which are in mutual involution and also commute with the energy, and then introduces a change of variables which has such functions among the generalized momenta, defined as follows. 
Assume that $\mathrm{C}^{(i)}$ is not parallel to $k^{(3)}$ and let

$$
n_{i}:=k^{(3)} \times \mathrm{C}^{(i)},
$$

so that $n_{i} \neq 0$ well defines the intersection ("node") of the $\left(k^{(1)}, k^{(2)}\right)$ plane with the plane orthogonal to $\mathrm{C}^{(i)}$ (the plane of the orbit ${ }^{6}$ ). Then define the following coordinates

$$
\left\{\begin{array} { l } 
{ \mathrm { H } _ { i } = \mathrm { C } _ { 3 } ^ { ( i ) } } \\
{ \mathrm { R } _ { i } : = \frac { y ^ { ( i ) } \cdot x ^ { ( i ) } } { | x ^ { ( i ) } | } } \\
{ \Phi _ { i } : = | \mathrm { C } ^ { ( i ) } | }
\end{array} \left\{\begin{array}{l}
\mathrm{h}_{i}=\alpha_{k^{(3)}}\left(k^{(1)}, n_{i}\right) \\
\mathrm{r}_{i}:=\left|x^{(i)}\right| \\
\varphi_{i}:=\alpha_{\mathrm{C}^{(i)}}\left(n_{i}, x^{(i)}\right) .
\end{array}\right.\right.
$$

Here, given three independent vectors $u, v, w \in \mathbb{R}^{3}$, with $w \perp(u, v), \alpha_{w}(u, v) \in \mathbb{T}$ denotes the oriented angle formed by $(u, v)$ in the positive (counterclockwise) verse determined by $w$.

The inverse formulae of (10) are as follows. If

$$
\bar{x}^{(i)}\left(\mathrm{R}_{i}, \Phi_{i}, \mathrm{r}_{i}, \varphi_{i}\right):=\left\{\begin{array}{c}
\mathrm{r}_{i} \cos \varphi_{i} \\
\mathrm{r}_{i} \sin \varphi_{i}
\end{array} \bar{y}^{(i)}\left(\mathrm{R}_{i}, \Phi_{i}, \mathrm{r}_{i}, \varphi_{i}\right):=\left\{\begin{array}{l}
\mathrm{R}_{i} \cos \varphi_{i}-\frac{\Phi_{i}}{\mathrm{r}_{i}} \sin \varphi_{i} \\
\mathrm{R}_{i} \sin \varphi_{i}+\frac{\Phi_{i}}{\mathrm{r}_{i}} \cos \varphi_{i}
\end{array}\right.\right.
$$

then

$$
\begin{aligned}
& x^{(i)}\left(\mathrm{R}_{i}, \Phi_{i}, \mathrm{r}_{i}, \varphi_{i}\right)=\mathrm{R}^{(3)}\left(\mathrm{h}_{i}\right) \mathrm{R}^{(1)}\left(\iota_{i}\right) \bar{x}^{(i)}\left(\mathrm{R}_{i}, \Phi_{i}, \mathrm{r}_{i}, \varphi_{i}\right) \\
& y^{(i)}\left(\mathrm{R}_{i}, \Phi_{i}, \mathrm{r}_{i}, \varphi_{i}\right)=\mathrm{R}^{(3)}\left(\mathrm{h}_{i}\right) \mathrm{R}^{(1)}\left(\iota_{i}\right) \bar{y}^{(i)}\left(\mathrm{R}_{i}, \Phi_{i}, \mathrm{r}_{i}, \varphi_{i}\right)
\end{aligned}
$$

where $\iota_{i}$ denote the convex angles between $\mathrm{C}$ and $\mathrm{C}^{(i)}$, determined by

$$
\cos \iota_{i}=\frac{\mathrm{H}_{i}}{\Phi_{i}}
$$

$\mathrm{R}^{(1)}(\iota), \mathrm{R}^{(3)}(\mathrm{h})$ are the elementary rotations

$$
\mathrm{R}^{(1)}(\iota)=\left(\begin{array}{ccc}
1 & 0 & 0 \\
0 & \cos i & -\sin i \\
0 & \sin i & \cos i
\end{array}\right) \quad \mathrm{R}^{(3)}(\mathrm{h})=\left(\begin{array}{ccc}
\cosh & -\sinh & 0 \\
\sinh & \cosh & 0 \\
0 & 0 & 1
\end{array}\right) .
$$

The change (10) is homogeneous and $h_{2 \mathrm{~b}}^{(i)}$ is transformed into

$$
h_{\mathrm{pc}}^{(i)}:=\frac{\mathrm{R}_{i}^{2}}{2 \mathfrak{m}_{i}}+\frac{\Phi_{i}^{2}}{2 m_{i} \mathrm{r}_{i}^{2}}-\frac{\mathfrak{M}_{i} \mathfrak{m}_{i}}{\mathrm{r}_{i}} .
$$

As expected, the angles $\mathrm{h}_{i}$ and $\varphi_{i}$ disappear from $h_{\mathrm{pc}}^{(i)}$ since they are conjugated to the integrals $\mathrm{H}_{i}, \Phi_{i}$. The first degeneracy of the problem consists in the fact that the action $\mathrm{H}_{i}$ disappears. This happens because its conjugated angle $\mathrm{h}_{i}$ is also an integral (it is again related to $\mathrm{C}^{(i)}$ ) .

We shall see in $\S 4.2$ that this "rotational" degeneracy of the two-body problem has a full generalization in the many-body case. This remarkable fact has been only recently pointed out [23], [8], [7].

The second step consists into the integration of the one-dimensional Hamiltonian $h_{\mathrm{pc}}^{(i)}$, via the Hamilton-Jacobi method.

\footnotetext{
${ }^{6} \mathrm{By}$ the conservation of $\mathrm{C}^{(i)}$, the orbit of $h_{2 \mathrm{~b}}^{(i)}$ lies in a plane.
} 
We are only interested to do it in the region of phase space where $h_{\mathrm{pc}}^{(i)}<0$, where the energy levels $h_{2 \mathrm{~b}}^{(i)}=\mathrm{E}_{2 \mathrm{~b}}^{(i)}$ are compact. In this case, one finds a two-dimensional canonical change variables parametrized by $\Phi_{i}$

$$
\left(\Lambda_{i}, \ell_{i}\right) \rightarrow\left(\mathrm{R}_{i}, \mathrm{r}_{i}\right)
$$

which integrates $h_{\mathrm{pc}}^{(i)}$. The integrated form of $h_{\mathrm{pc}}^{(i)}$ is just the "Keplerian" one

$$
h_{\mathrm{K}}^{(i)}=-\frac{\mathfrak{m}_{i}^{3} \mathfrak{M}_{i}^{2}}{2 \Lambda_{i}^{2}} .
$$

As expected, the angles $\left(\ell_{i}, \mathrm{~g}_{i}\right)$ disappear since they are conjugated to the integrals $\left(\Lambda_{i}, \Gamma_{i}\right)$. The fact that also $\Gamma_{i}$ disappears (this is the second degeneracy of the problem) is related to the integral $\mathrm{L}_{i}$ : the angle $\mathrm{g}_{i}$, conjugated to $\Gamma_{i}$, just corresponds to be the angle $\widehat{n_{i} \mathrm{~L}_{i}}$, and this angle does not move, so also $\Gamma_{i}$ disappears.

The motion then reduces to the "third Kepler law" for the angle $\ell_{i}$

$$
\ell_{i} \rightarrow \ell_{i}+\frac{\mathfrak{m}_{i}^{3} \mathfrak{M}_{i}^{2}}{\Lambda_{i}^{3}} t
$$

while all the remaining coordinates remain constant.

Let us recall that the change (15) can be extended to a four-dimensional canonical change

$$
\left(\Lambda_{i}, \Gamma_{i}, \ell_{i}, \mathrm{~g}_{i}\right) \rightarrow\left(\mathrm{R}_{i}, \Phi_{i}, \mathrm{r}_{i}, \varphi_{i}\right) \quad \text { with } \quad \Gamma_{i}=\Phi_{i}
$$

involving the four coordinates $\left(\Lambda_{i}, \Gamma_{i}, \ell_{i}, \mathrm{~g}_{i}\right) \in \mathbb{R}^{2} \times \mathbb{T}^{2}$. Taking in count also the couples $\left(\mathrm{H}_{i}, \mathrm{~h}_{i}\right)$, one lifts this map to a six-dimensional one

$$
\left(\Lambda_{i}, \Gamma_{i}, \mathrm{H}_{i}, \ell_{i}, \mathrm{~g}_{i}, \mathrm{~h}_{i}\right) \rightarrow\left(\mathrm{R}_{i}, \Phi_{i}, \mathrm{H}_{i}, \mathrm{r}_{i}, \varphi_{i}, \mathrm{~h}_{i},\right) \quad \text { with } \quad \Gamma_{i}=\Phi_{i}
$$

via the identity on such couples.

In count of relations (11) and (12), we correspondingly have two canonical maps

$$
\begin{array}{ll}
\phi_{\mathrm{D}}^{(i)}: & \left(\Lambda_{i}, \Gamma_{i}, \mathrm{H}_{i}, \ell_{i}, \mathrm{~g}_{i}, \mathrm{~h}_{i}\right) \rightarrow\left(y_{\mathrm{D}}^{(i)}, x_{\mathrm{D}}^{(i)}\right) \in \mathbb{R}^{3} \times \mathbb{R}^{3} \\
\bar{\phi}_{\mathrm{D}}^{(i)}: & \left(\Lambda_{i}, \Gamma_{i}, \ell_{i}, \mathrm{~g}_{i}\right) \rightarrow\left(\bar{y}_{\mathrm{D}}^{(i)}, \bar{x}_{\mathrm{D}}^{(i)}\right) \in \mathbb{R}^{2} \times \mathbb{R}^{2}
\end{array}
$$

which we shall refer to as "spatial", "planar" Delaunay map, respectively. By similarity, we might call the map (15) "linear" Delaunay map.

The planar and the spatial maps are related via

$$
\begin{aligned}
& y_{\mathrm{D}}^{(i)}\left(\Lambda_{i}, \Gamma_{i}, \mathrm{H}_{i}, \ell_{i}, \mathrm{~g}_{i}, \mathrm{~h}_{i}\right)=\mathrm{R}^{(3)}\left(\mathrm{h}_{i}\right) \mathrm{R}^{(1)}\left(\iota_{i}\right) \bar{y}_{\mathrm{D}}^{(i)}\left(\Lambda_{i}, \Gamma_{i}, \ell_{i}, \mathrm{~g}_{i}\right) \\
& x_{\mathrm{D}}^{(i)}\left(\Lambda_{i}, \Gamma_{i}, \mathrm{H}_{i}, \ell_{i}, \mathrm{~g}_{i}, \mathrm{~h}_{i}\right)=\mathrm{R}^{(3)}\left(\mathrm{h}_{i}\right) \mathrm{R}^{(1)}\left(\iota_{i}\right) \bar{x}_{\mathrm{D}}^{(i)}\left(\Lambda_{i}, \Gamma_{i}, \ell_{i}, \mathrm{~g}_{i}\right)
\end{aligned}
$$

with $\cos \iota_{i}=\frac{\mathrm{H}_{i}}{\Gamma_{i}}$.

We recall that relations $y^{(i)}=\mathfrak{m}_{i} \dot{x}^{(i)}$ and (17) imply that $x^{(i)}$ and $y^{(i)}$ in (20) (as well as $\bar{x}_{\mathrm{D}}^{(i)}$ and $\bar{y}_{\mathrm{D}}^{(i)}$, or $\mathrm{r}_{i}$ and $\mathrm{R}_{i}$ ) are related by

$$
y^{(i)}\left(\Lambda_{i}, \Gamma_{i}, \mathrm{H}_{i}, \ell_{i}, \mathrm{~g}_{i}, \mathrm{~h}_{i}\right)=\frac{\mathfrak{m}_{i}^{4} \mathfrak{M}_{i}^{2}}{\Lambda_{i}^{3}} \partial_{\ell_{i}} x^{(i)}\left(\Lambda_{i}, \Gamma_{i}, \mathrm{H}_{i}, \ell_{i}, \mathrm{~g}_{i}, \mathrm{~h}_{i}\right)
$$

Let us now describe the meaning of the four variables at l. h. s. of (18).

As well known, the pull back orbits $t \rightarrow\left(y^{(i)}(t), x^{(i)}(t)\right)$ are ellipses $\mathfrak{E}_{i}$ with a focus in the origin. If $P^{(i)}, a^{(i)}, e^{(i)}$ denote the perihelion, the semi-major axis, the eccentricity of $\mathfrak{E}_{i}, \mathcal{A}^{(i)}$ the area 
spanned from $P^{(i)}$ to $x^{(i)}, \mathcal{A}^{(i)}=\pi\left(a^{(i)}\right)^{2} \sqrt{1-\left(e^{(i)}\right)^{2}}$ the total area of $\mathfrak{E}_{i}$, then $P^{(i)}$ has the direction of $\mathrm{L}_{i}$ and the coordinates at left hand side in (18) are defined by

$$
\left\{\begin{array} { l } 
{ \Lambda _ { i } = \mathfrak { m } _ { i } \sqrt { \mathfrak { M } _ { i } a ^ { ( i ) } } } \\
{ \Gamma _ { i } = \Phi _ { i } = | \mathrm { C } ^ { ( i ) } | }
\end{array} \quad \left\{\begin{array}{l}
\ell_{i}=2 \pi \frac{\mathcal{A}^{(i)}}{\mathcal{A}_{\mathrm{tot}}^{(i)}} \\
\mathrm{g}_{i}=\alpha_{\mathrm{C}^{(i)}}\left(n_{i}, P^{(i)}\right)
\end{array}\right.\right.
$$

The angles $\ell_{i}, \mathrm{~g}_{i}$ in this formula and $\mathrm{h}_{i}$ in (10) are called "mean anomaly", "argument of perihelion" and "longitude of the node".

Delaunay coordinates are not defined when $\mathrm{C}^{(i)} \| k^{(3)}$ or the eccentricity of the $i^{\text {th }}$ ellipse is zero. Then, following Poincaré, one modifies them by considering the canonical variables

$$
\Lambda_{i}, \lambda_{i}, \eta_{i}, \xi_{i}, \mathrm{p}_{i}, \mathrm{q}_{i}
$$

where $\Lambda_{i}$ are as above, and

$$
\lambda_{i}=\ell_{i}+\mathrm{g}_{i}+\mathrm{h}_{i} \quad\left\{\begin{array} { l } 
{ \eta _ { i } = \sqrt { 2 ( \Lambda _ { i } - \Gamma _ { i } ) } \operatorname { c o s } ( \mathrm { g } _ { i } + \mathrm { h } _ { i } ) } \\
{ \xi _ { i } = - \sqrt { 2 ( \Lambda _ { i } - \Gamma _ { i } ) } \operatorname { s i n } ( \mathrm { g } _ { i } + \mathrm { h } _ { i } ) }
\end{array} \quad \left\{\begin{array}{l}
\mathrm{p}_{i}=\sqrt{2\left(\Gamma_{i}-\mathrm{H}_{i}\right)} \cos \left(\mathrm{h}_{i}\right) \\
\mathrm{q}_{i}=-\sqrt{2\left(\Gamma_{i}-\mathrm{H}\right)} \sin \left(\mathrm{h}_{i}\right) .
\end{array}\right.\right.
$$

One lets $\mathrm{z}_{i}:=\left(\eta_{i}, \xi_{i}, \mathrm{p}_{i}, \mathrm{q}_{i}\right)$ and $\mathrm{z}=\left(\mathrm{z}_{1}, \cdots, \mathrm{z}_{n}\right)$. The Poincaré maps

$$
\phi_{\mathrm{P}}^{(i)}: \quad\left(\Lambda_{i}, \lambda_{i}, \eta_{i}, \xi_{i}, \mathrm{p}_{i}, \mathrm{q}_{i}\right) \rightarrow\left(y^{(i)}, x^{(i)}\right)
$$

turn out to be regular also for $\left(\eta_{i}, \xi_{i}\right)=0$ or $\left(\mathrm{p}_{i}, \mathrm{q}_{i}\right)=0$, which correspond to the vanishing of eccentricities and inclinations. They have the form

$$
y^{(i)}=\mathfrak{R}_{\mathrm{P}}^{(i)}\left(\Lambda_{i}, \mathrm{z}_{i}\right) \bar{x}_{\mathrm{P}}^{(i)}\left(\Lambda_{i}, \lambda_{i}, \eta_{i}, \xi_{i}\right) \quad x^{(i)}=\mathfrak{R}_{\mathrm{P}}^{(i)}\left(\Lambda_{i}, \mathrm{z}_{i}\right) \bar{x}_{\mathrm{P}}^{(i)}\left(\Lambda_{i}, \lambda_{i}, \eta_{i}, \xi_{i}\right)
$$

where $\mathfrak{R}_{\mathrm{P}}^{(i)}\left(\Lambda_{i}, \mathrm{z}_{i}\right)$ are unitary $3 \times 3$ matrices depending only on $\left(\Lambda_{i}, \mathrm{z}_{i}\right)$ and reducing to the identity for $\left(\mathrm{p}_{i}, \mathrm{q}_{i}\right)=0$ and

$$
\bar{\phi}_{\mathrm{P}}^{(i)}: \quad\left(\Lambda_{i}, \lambda_{i}, \eta_{i}, \xi_{i}\right) \rightarrow\left(\bar{x}_{\mathrm{P}}^{(i)}\left(\Lambda_{i}, \lambda_{i}, \eta_{i}, \xi_{i}\right), \bar{y}_{\mathrm{P}}^{(i)}\left(\Lambda_{i}, \lambda_{i}, \eta_{i}, \xi_{i}\right)\right)
$$

are the so-called planar Poincaré maps.

\section{$3.2 \quad$ Kepler maps}

Delaunay and Poincaré maps put the unperturbed term in (2) in the form

$$
h_{\mathrm{K}}(\Lambda)=-\sum_{1 \leq i \leq n} \frac{\mathfrak{m}_{i}^{3} \mathfrak{M}_{i}^{2}}{2 \Lambda_{i}^{2}}
$$

Despite of this good aspect, however, they do not take in account the reduction of the integral $\mathrm{C}$ in (8). Namely, the transformed Hamiltonians ${ }^{7}$

$$
\mathrm{H}_{\mathrm{D}}:=\mathrm{H}_{\mathrm{hel}} \circ \phi_{\mathrm{D}}:=\mathrm{h}_{\mathrm{K}}(\Lambda)+\mu f_{\mathrm{D}}(\Lambda, \Gamma, \mathrm{H}, \ell, \mathrm{g}, \mathrm{h})
$$

\footnotetext{
7 If $\Lambda=\left(\Lambda_{1}, \cdots, \Lambda_{n}\right), \Gamma=\left(\Gamma_{1}, \cdots, \Gamma_{n}\right), \mathrm{H}=\left(\mathrm{H}_{1}, \cdots, \mathrm{H}_{n}\right), \ell=\left(\ell_{1}, \cdots, \ell_{n}\right), \mathrm{g}=\left(\mathrm{g}_{1}, \cdots, \mathrm{g}_{n}\right), \mathrm{h}=$ $\left(\mathrm{h}_{1}, \cdots, \mathrm{h}_{n}\right)$ denote the collection of all Delaunay coordinates, we shall denote as

$$
\phi_{\mathrm{D}}: \quad(\Lambda, \Gamma, \mathrm{H}, \ell, \mathrm{g}, \mathrm{h}) \rightarrow(y, x) \in \mathbb{R}^{3} \times \mathbb{R}^{3} \quad 1 \leq i \leq n
$$
}

the collection of all such maps. $\phi_{\mathrm{P}}$ is defined analogously. 
and

$$
\mathrm{H}_{\mathrm{P}}:=\mathrm{H}_{\text {hel }} \circ \phi_{\mathrm{P}}:=\mathrm{h}_{\mathrm{K}}(\Lambda)+\mu f_{\mathrm{P}}(\Lambda, \lambda, \mathrm{z})
$$

have $3 n$ degrees of freedom as well as $\mathrm{H}_{\text {hel }}$.

At this respect, we introduced in [25] the notion of "Kepler map". This is a generalization of Delaunay and Poincaré maps $\phi_{\mathrm{D}}$ and $\phi_{\mathrm{P}}$, motivated by the fact that the proper degeneracies of $h_{\mathrm{K}}$ provide, as mentioned in the introduction, more freedom in the choice of canonical coordinates for the planetary system.

- Given $2 n$ positive "mass parameters" $\mathfrak{m}_{1}, \cdots, \mathfrak{m}_{n}, \mathfrak{M}_{1}, \cdots, \mathfrak{M}_{n}$, a set $\mathfrak{X} \subset \mathbb{R}^{5 n}$ and a bijection

$$
\begin{aligned}
\tau: \mathfrak{X} & \rightarrow\left\{\left(\mathfrak{E}_{1}, \cdots, \mathfrak{E}_{n}\right) \in\left(E^{3}\right)^{n}, \mathfrak{E}_{i}: \text { ellipse }\right\} \\
\mathrm{X} \in \mathfrak{X} & \rightarrow\left(\mathfrak{E}_{1}(\mathrm{X}), \cdots, \mathfrak{E}_{n}(\mathrm{X})\right)
\end{aligned}
$$

which assigns to any $\mathrm{X} \in \mathfrak{X}$ an ordered set of $n$ ellipses $\left(\mathfrak{E}_{1}, \cdots, \mathfrak{E}_{n}\right)$ in the Euclidean space $E^{3}$ with strictly positive eccentricities and having a common focus $\mathrm{S}$, we shall say that an injective map

$$
\phi: \quad(\mathrm{X}, \ell) \in \mathcal{D}^{6 n}:=\mathfrak{X} \times \mathbb{T}^{n} \rightarrow\left(y_{\phi}(\mathrm{X}, \ell), x_{\phi}(\mathrm{X}, \ell)\right) \in\left(\mathbb{R}^{3}\right)^{n} \times\left(\mathbb{R}^{3}\right)^{n}
$$

is a Kepler map if $\phi$ associates to $(\mathrm{X}, \ell) \in \mathfrak{X} \times \mathbb{T}^{n}$, with $\ell=\left(\ell_{1}, \cdots, \ell_{n}\right)$ an element

$$
\left(y_{\phi}(\mathrm{X}, \ell), x_{\phi}(\mathrm{X}, \ell)\right)=\left(y_{\phi}^{(1)}\left(\mathrm{X}, \ell_{1}\right), \cdots, y_{\phi}^{(n)}\left(\mathrm{X}, \ell_{n}\right), x_{\phi}^{(1)}\left(\mathrm{X}, \ell_{1}\right), \cdots, x_{\phi}^{(n)}\left(\mathrm{X}, \ell_{n}\right)\right)
$$

in the following way. Letting, respectively, $P_{\phi}^{(i)}(\mathrm{X}), a_{\phi}^{(i)}(\mathrm{X}), e_{\phi}^{(i)}(\mathrm{X})$ and $N_{\phi}^{(i)}(\mathrm{X})$ the direction from $\mathrm{S}$ to the perihelion, the semi-major axis, the eccentricity and a prefixed direction of the plane of $\mathfrak{E}_{i}(\mathrm{X}), x_{\phi}^{(i)}\left(\mathrm{X}, \ell_{i}\right)$ are the coordinates with respect to a prefixed orthonormal frame $(i, j, k)$ centered in $\mathrm{S}$ of the point of $\mathfrak{E}_{i}(\mathrm{X})$ such that

$$
\mathcal{A}\left(x_{\phi}^{(i)}\left(\mathrm{X}, \ell_{i}\right)\right)=\frac{1}{2} a_{\phi}^{(i)} \sqrt{1-\left(e_{\phi}^{(i)}\right)^{2}} \ell_{i} \quad \bmod \pi a_{\phi}^{(i)} \sqrt{1-\left(e_{\phi}^{(i)}\right)^{2}}
$$

is the area spanned from $P_{\phi}^{(i)}(\mathrm{X})$ to $x_{\phi}^{(i)}\left(\mathrm{X}, \ell_{i}\right)$ relatively to the positive (counterclockwise) orientation determined by $N_{\phi}^{(i)}(\mathrm{X})$ and

$$
y_{\phi}^{(i)}\left(\mathrm{X}, \ell_{i}\right)=\mathfrak{m}_{i} \sqrt{\frac{\mathfrak{M}_{i}}{\left(a^{(i)}\right)^{3}}} \partial_{\ell_{i}} x_{\phi}^{(i)}\left(\mathrm{X}, \ell_{i}\right) .
$$

- One can consider canonical Kepler maps, i.e., such that $\mathrm{X} \in \mathfrak{X}$ has the form $\mathrm{X}=(\mathrm{P}, \mathrm{Q}, \Lambda)$ where $\Lambda=\left(\Lambda_{1}, \cdots, \Lambda_{n}\right)=\left(\mathfrak{m}_{1} \sqrt{\mathfrak{M}_{1} a_{\phi}^{(1)}}, \cdots, \mathfrak{m}_{n} \sqrt{\mathfrak{M}_{n} a_{\phi}^{(n)}}\right), \mathrm{P}=\left(\mathrm{P}_{1}, \cdots, \mathrm{P}_{2 n}\right), \mathrm{Q}=$ $\left(\mathrm{Q}_{1}, \cdots, \mathrm{Q}_{2 n}\right)$ and the map

$$
(\Lambda, \ell, \mathrm{P}, \mathrm{Q}) \rightarrow(y, x)=\left(y^{(1)}, \cdots, y^{(n)}, x^{(1)}, \cdots, x^{(n)}\right)
$$

preserves the standard 2-form:

$$
\sum_{1 \leq i \leq n} d \Lambda_{i} \wedge d \ell_{i}+\sum_{1 \leq i \leq 2 n} d \mathrm{P}_{i} \wedge d \mathrm{Q}_{i}=\sum_{1 \leq i \leq n} \sum_{1 \leq j \leq 3} d y_{j}^{(i)} \wedge d x_{j}^{(i)}
$$

or, equivalently,

$$
\sum_{1 \leq i \leq 2 n} d \mathrm{P}_{i} \wedge d \mathrm{Q}_{i}=\sum_{1 \leq i \leq n} d \Gamma_{i} \wedge d \mathrm{~g}_{i}+\sum_{1 \leq i \leq n} d \mathrm{H}_{i} \wedge d \mathrm{~h}_{i}
$$


- One can also consider canonical Kepler maps on manifolds, i.e., maps

$$
(\Lambda, \ell, \overline{\mathrm{P}}, \overline{\mathrm{Q}}) \rightarrow(y, x)
$$

where $\overline{\mathrm{P}}=\left(\overline{\mathrm{P}}_{1}, \cdots, \overline{\mathrm{P}}_{m}\right), \overline{\mathrm{Q}}=\left(\overline{\mathrm{Q}}_{1}, \cdots, \overline{\mathrm{Q}}_{m}\right)$, with $m \leq 2 n$ and such that (26) holds with $\sum_{1 \leq i \leq 2 n} d \mathrm{P}_{i} \wedge d \mathrm{Q}_{i}$ replaced by $\sum_{1 \leq i \leq m} d \overline{\mathrm{P}}_{i} \wedge d \overline{\mathrm{Q}}_{i}$.

Common properties to Kepler maps, with special attention to their application to the planetary hamiltonian (e.g., in the heliocentric form (2)) have been shortly discussed in [25]. Here we just recall two facts.

- Relations (24) and (25) imply that the two-body Hamiltonian (7) becomes

$$
h_{\mathrm{K}}^{(i)}=-\frac{\mathfrak{m}_{i} \mathfrak{M}_{i}}{2 a_{\phi}^{(i)}} .
$$

For a canonical map, one has just (16).

- Due to (25) and to equation

$$
\sqrt{\frac{\mathfrak{M}_{i}}{\left(a_{\phi}^{(i)}\right)^{3}}} \partial_{\ell_{i}} y_{\phi}^{(i)}\left(\mathrm{X}, \ell_{i}\right)=-\mathfrak{m}_{i} \mathfrak{M}_{i} \frac{x_{\phi}^{(i)}\left(\mathrm{X}, \ell_{i}\right)}{\left|x_{\phi}^{(i)}\left(\mathrm{X}, \ell_{i}\right)\right|^{3}}
$$

(which follows from (25) and the definition of $x_{\phi}^{(i)}\left(\mathrm{X}, \ell_{i}\right)$ ), one has

$$
\int_{\mathbb{T}} \frac{x_{\phi}^{(i)}\left(\mathrm{X}, \ell_{i}\right)}{\left|x_{\phi}^{(i)}\left(\mathrm{X}, \ell_{i}\right)\right|^{3}} d \ell_{i}=0 \quad \int_{\mathbb{T}} y_{\phi}^{(i)}\left(\mathrm{X}, \ell_{i}\right) d \ell_{i}=0 .
$$

Such relations give us the opportunity of noticing a dynamical equivalence between the heliocentric, Jacobi reduction, announced in $§ 2$, which is explained in the following section.

\subsection{Dynamical equivalence of the heliocentric and Jacobi reduction}

We just consider the case $n=2$. Let $\phi$ be a given Kepler map in correspondence of mass parameters $\left(\mathfrak{m}_{i}, \mathfrak{M}_{i}\right)_{1 \leq i \leq 2}$ and $\tilde{\phi}$ the same Kepler map in correspondence of $\left(\tilde{\mathfrak{m}}_{i}, \tilde{\mathfrak{M}}_{i}\right)_{1 \leq i \leq 2}$. Here, $\left(\mathfrak{m}_{i}, \mathfrak{M}_{i}\right)_{1 \leq i \leq 2}$ and $\left(\tilde{\mathfrak{m}}_{i}, \tilde{\mathfrak{M}}_{i}\right)_{1 \leq i \leq 2}$ are as in $\S 2$. Let $f_{\text {hel }, \phi}:=f_{\text {hel }} \circ \phi, f_{\text {Jac }, \tilde{\phi}}:=f_{\text {Jac }} \circ \tilde{\phi}$ and put

$$
f_{\mathrm{hel}, \phi}^{\mathrm{av}}:=\frac{1}{(2 \pi)^{2}} \int_{\mathbb{T}^{2}} f_{\mathrm{hel}, \phi} d \ell_{1} d \ell_{2} \quad f_{\mathrm{Jac}, \tilde{\phi}}^{\mathrm{av}}:=\frac{1}{(2 \pi)^{2}} \int_{\mathbb{T}^{2}} f_{\mathrm{Jac}, \tilde{\phi}} d \ell_{1} d \ell_{2} .
$$

Then relations (27) immediately imply ${ }^{8}$

$$
f_{\mathrm{hel}, \phi}^{\mathrm{av}}=f_{\mathrm{Jac}, \tilde{\phi}}^{\mathrm{av}}+\mathrm{O}(\mu),
$$

with the common value of the two integrals being just the average of the Newtonian term, $-\frac{m_{1} m_{2}}{(2 \pi)^{2}} \int_{\mathbb{T}^{2}} \frac{d \ell_{1} d \ell_{2}}{\left|x_{\phi}^{(1)}-x_{\phi}^{(2)}\right|}+\mathrm{O}(\mu)$. This relation is important in view of applications to KAM theory; for example, in relation to the proof of Arnold's Theorem, it says that the Birkhoff invariants that one finds using any of the two reductions differ ${ }^{9}$ just by $\mathrm{O}(\mu)$.

\footnotetext{
${ }^{8}$ Recall $(5)$ and $\mathfrak{m}_{i}=m_{i}+\mathrm{O}(\mu)=\tilde{\mathfrak{m}}_{i}, \mathfrak{M}_{i}=m_{0}+\mathrm{O}(\mu)=\tilde{\mathfrak{M}}_{i}$. Note that (28) holds also taking the integral only with respect to $\ell_{2}$.

${ }^{9}$ Observe the following remarkable consequence of this, noticed in [1]: while the first order Birkhoff invariants $\Omega_{i}$ associated to $f_{\text {hel, } \phi}^{\text {av }}$ satisfy identically Herman resonance $\sum_{i} \Omega_{i} \equiv 0$, this resonance is instead verified only at the lowest order in $\mu$ by the invariants associated to $f_{\mathrm{Jac}, \tilde{\phi}}^{\text {av }}$.
} 
Equality (28) has a dynamical explanation.

Averaging theory states that, for a properly-degenerate dynamical system

$$
\mathrm{H}(I, \varphi, u, v)=h(I)+\mu f(I, \varphi, u, v)
$$

there exists an associated "secular" system

$$
\mathrm{H}^{\sec }(I, \varphi, u, v)=h(I)+\mu f^{\mathrm{av}}(I, u, v)+\mathrm{O}\left(\mu^{2}\right)
$$

with $f^{\text {av }}(I, u, v)=\frac{1}{(2 \pi)^{n}} \int_{\mathbb{T}^{n}} f(I, \varphi, u, v) d \varphi$, related to $\mathrm{H}(I, \varphi, u, v)$ via a symplectic, close to the identity transformation. We recall that the transformation realizing this conjugation needs not to be unique, but $\mathrm{H}^{\text {sec }}$ is uniquely determined up to $\mathrm{O}\left(\mu^{2}\right)$ if such transformation is chosen in the class of symplectic, $\mu$-close to the identity ones.

Then one can conjugate the two Hamiltinians

$$
\mathrm{H}_{\mathrm{hel}, \phi}:=\mathrm{H}_{\mathrm{hel}} \circ \phi=-\sum_{1 \leq i \leq 2} \frac{\mathfrak{m}_{i}^{3} \mathfrak{M}_{i}^{2}}{2 \Lambda_{i}^{2}}+\mu f_{\mathrm{hel}, \phi}(\Lambda, \ell, P, Q)
$$

and

$$
\tilde{\mathrm{H}}_{\mathrm{Jac}, \tilde{\phi}}:=\mathrm{H}_{\mathrm{Jac}} \circ \tilde{\phi}=-\sum_{1 \leq i \leq 2} \frac{\tilde{\mathfrak{m}}_{i}^{3} \tilde{\mathfrak{M}}_{i}^{2}}{2 \Lambda_{i}^{2}}+\mu f_{\mathrm{Jac}, \tilde{\phi}}(\Lambda, \ell, P, Q)
$$

respectively to

$$
\begin{gathered}
\mathrm{H}_{\mathrm{hel}, \phi}^{\mathrm{sec}}=-\sum_{1 \leq i \leq 2} \frac{\mathfrak{m}_{i}^{3} \mathfrak{M}_{i}^{2}}{2 \Lambda_{i}^{2}}+\mu f_{\mathrm{hel}, \phi}^{\mathrm{av}}(\Lambda, P, Q)+\mathrm{O}\left(\mu^{2}\right) \\
\tilde{\mathrm{H}}_{\mathrm{Jac}, \tilde{\phi}}^{\mathrm{sec}}=-\sum_{1 \leq i \leq 2} \frac{\tilde{\mathfrak{m}}_{i}^{3} \tilde{\mathfrak{M}}_{i}^{2}}{2 \Lambda_{i}^{2}}+\mu f_{\mathrm{Jac}, \tilde{\phi}}^{\mathrm{av}}(\Lambda, P, Q)+\mathrm{O}\left(\mu^{2}\right) .
\end{gathered}
$$

Furthermore, relation (6) implies that $\mathrm{H}_{\mathrm{hel}, \phi}^{\mathrm{sec}}$ and $\tilde{\mathrm{H}}_{\mathrm{Jac}, \tilde{\phi}}^{\mathrm{sec}}$ are related by a symplectic, close to the identity transformation. Using finally ${ }^{10}$

$$
\mathfrak{m}_{i}^{3} \mathfrak{M}_{i}^{2}=\tilde{\mathfrak{m}}_{i}^{3} \tilde{\mathfrak{M}}_{i}^{2}+\mathrm{O}\left(\mu^{2}\right)
$$

and the uniqueness of the secular system associated to any of the two $\mathrm{H}_{\mathrm{hel}, \phi}$ and $\tilde{\mathrm{H}}_{\mathrm{Jac}, \tilde{\phi}}$ we have (28).

\section{Examples of canonical Kepler maps}

The first classical example of Kepler map is clearly given by the Delaunay, Poincaré maps. In these cases, relations (24) and (25) are a consequence of the fact that such maps are canonical modifications of the six-dimensional map (19).

On the other hand, in order that (24) and (25) are satisfied, it is not necessary to modify (19). Starting from the planar map (18) or even the linear one (15) is sufficient.

In $\S 4.1-\S 4.3$ we present examples of Kepler maps which are based on the planar map (18); in $\S 4.5$ we provide an example based on the linear map (15).

${ }^{10}$ This follows from

$$
\mathfrak{m}_{1}=\tilde{\mathfrak{m}}_{1}, \quad \mathfrak{m}_{2}=\tilde{\mathfrak{m}}_{2}+\mathrm{O}\left(\mu^{2}\right), \quad \mathfrak{m}_{i} \mathfrak{M}_{i}=\tilde{\mathfrak{m}}_{i} \tilde{\mathfrak{M}}_{i}=m_{0} m_{i}
$$

(The relation between $\mathfrak{m}_{2}$ and $\tilde{\mathfrak{m}}_{2}$ deserves to be remarked.) 


\subsection{Jacobi-Radau's reduction of the nodes for three bodies}

The "Jacobi reduction of the nodes" is a classical tool for reducing the number of degrees of freedom of (2) from six to four, in the case $n=2$. Its main properties are

- it works only for the spatial problem (its planar limit is singular);

- uses the planar Delaunay maps (18);

- it can be regarded as an example of Kepler map on a eight-dimensional manifold (compare (34) below).

Let us recall some history.

In 1842, Jacobi [17] discovered that (after the linear momentum reduction) the twelve differential equations of the three-body problem dynamical system might be reduced to a system of seven of equations: six of them of the first order, one of the second order. Essentially, four degrees of freedom. Clearly, what boils down is the reduction of the angular momentum integral.

Jacobi's procedure inherited a Hamiltonian aspect after the paper by Radau [26], that now we explain. Let

$$
\begin{aligned}
\mathrm{H}_{3 \mathrm{~b}}(\mathrm{R}, \Phi, \mathrm{H}, \mathrm{r}, \varphi, \mathrm{h}) & =\frac{\mathrm{R}_{1}^{2}}{2 \mathfrak{m}_{1}}+\frac{\Phi_{1}^{2}}{2 \mathfrak{m}_{1} \mathrm{r}_{1}^{2}}-\frac{\mathfrak{m}_{1} \mathfrak{M}_{1}}{\mathrm{r}_{1}}+\frac{\mathrm{R}_{2}^{2}}{2 \mathfrak{m}_{2}}+\frac{\Phi_{2}^{2}}{2 \mathfrak{m}_{2} \mathrm{r}_{2}^{2}}-\frac{\mathfrak{m}_{2} \mathfrak{M}_{2}}{\mathrm{r}_{2}} \\
& +\mu\left(\frac{y^{(1)} \cdot y^{(2)}}{m_{0}}-\frac{m_{1} m_{2}}{\left|x^{(1)}-x^{(2)}\right|}\right)(\mathrm{R}, \Phi, \mathrm{H}, \mathrm{r}, \varphi, \mathrm{h})
\end{aligned}
$$

be the heliocentric, three-body Hamiltonian for $n=2$ planets, written in the coordinates (10).

Recall that one has, for the heliocentric coordinates, the expressions in (12). Such relations and invariance by rotations of $\mathrm{H}_{3 \mathrm{~b}}$ imply that $\mathrm{H}_{3 \mathrm{~b}}$ depends upon the angles $\mathrm{h}_{1}$ and $\mathrm{h}_{2}$ only via the difference $\mathrm{h}_{1}-\mathrm{h}_{2}$. If we fix (as it is always possible to do) the initial frame $\mathrm{F}_{0}=\left(k^{(1)}, k^{(2)}, k^{(3)}\right)$ such in a way that the total angular momentum $\mathrm{C}=\mathrm{C}^{(1)}+\mathrm{C}^{(2)}$ coincides with the $k^{(3)}$-axis, by definition, the nodes $n_{1}, n_{2}$ in (9) are opposite one to the other, as it follows from

$$
0=k^{(3)} \times \mathrm{C}=k^{(3)} \times \mathrm{C}^{(1)}+k^{(3)} \times \mathrm{C}^{(2)}=n_{1}+n_{2} .
$$

We then have

$$
\mathrm{h}_{2}-\mathrm{h}_{1}=\pi \quad \bmod 2 \pi .
$$

By this relation and again invariance by rotations of $\mathrm{H}_{3 \mathrm{~b}}$, replacing Eq. (12) into $\mathrm{H}_{3 \mathrm{~b}}$ (29) is equivalent to replace

$$
\begin{aligned}
& x^{(1)}=\mathrm{R}_{1}\left(\iota_{1}\right) \bar{x}^{(1)}\left(\mathrm{R}_{1}, \Phi_{1}, \mathrm{r}_{1}, \varphi_{1}\right) \\
& x^{(2)}=\mathrm{R}_{1}\left(-\iota_{2}\right) \bar{x}^{(2)}\left(\mathrm{R}_{2}, \Phi_{2}, \mathrm{r}_{2}, \varphi_{2}\right)
\end{aligned}
$$

and, similarly,

$$
\begin{aligned}
& y^{(1)}=\mathrm{R}_{1}\left(\iota_{1}\right) \bar{y}^{(1)}\left(\mathrm{R}_{1}, \Phi_{1}, \mathrm{r}_{1}, \varphi_{1}\right) \\
& y^{(2)}=\mathrm{R}_{1}\left(-\iota_{2}\right) \bar{y}^{(2)}\left(\mathrm{R}_{2}, \Phi_{2}, \mathrm{r}_{2}, \varphi_{2}\right) .
\end{aligned}
$$

Here we have used $\mathrm{R}^{(3)}(\pi) \mathrm{R}^{(1)}(\iota)=\mathrm{R}^{(1)}(-\iota) \mathrm{R}^{(3)}(\pi)$ and an inessential shift of $\varphi_{2}$ by $\pi$. Fix the eight-dimensional ${ }^{11}$ manifold

$$
\mathcal{M}_{n_{1}}=\left\{k^{(1)}=n_{1}, \quad k^{(3)}=\mathrm{C}\right\} .
$$

\footnotetext{
${ }^{11}$ Equation $k^{(3)}=\mathrm{C}$ is equivalent to $\mathrm{C}_{1}=\mathrm{C}_{2}=0$, hence corresponds to two conditions. Equation $k^{(1)}=n_{1}$ also is bi-dimensional, since, in general, $k^{(1)}$ varies among vectors verifying $k^{(1)} \perp k^{(3)}=\mathrm{C}\left(\perp n_{1}\right)$. We then have four independent conditions in a 12-dimensional phase space.
} 
This corresponds to fix a rotating frame about $\mathrm{C}$ such that, with respect to it, node of the plane perpendicular to $\mathrm{C}^{(1)}$ is fixed. The aim is to find canonical coordinates for $\mathcal{M}_{n_{1}}$. If we let ${ }^{12}$ $\mathrm{G}:=|\mathrm{C}|$, we may also write the respective inclinations $\iota_{1}$ and $\iota_{2}$ as

$$
\cos \iota_{1}=\frac{\Phi_{1}^{2}+\mathrm{G}^{2}-\Phi_{2}^{2}}{2 \Phi_{1} \mathrm{G}} \quad \cos \iota_{2}=\frac{\Phi_{2}^{2}+\mathrm{G}^{2}-\Phi_{1}^{2}}{2 \Phi_{2} \mathrm{G}} .
$$

These relations follow considering the triangle formed by $\mathrm{C}^{(1)}, \mathrm{C}^{(2)}$ and $\mathrm{C}$. Similarly, the convex angle $\iota=\pi-\left(\iota_{1}+\iota_{2}\right)$ formed by $\mathrm{C}^{(1)}$ and $\mathrm{C}^{(2)}$ is determined by

$$
\cos \iota=\frac{\Phi_{1}^{2}+\Phi_{2}^{2}-\mathrm{G}^{2}}{2 \Phi_{1} \Phi_{2}} .
$$

Again, invariance by rotations of $\mathrm{H}_{3 \mathrm{~b}}$ implies that only this angle is needed.

Let

$$
\begin{aligned}
\mathrm{H}_{3 \mathrm{~b}, \text { red }} & =\sum_{1 \leq i \leq 2}\left(\frac{\mathrm{R}_{i}^{2}}{2 \mathfrak{m}_{i}}+\frac{\Phi_{i}^{2}}{2 \mathfrak{m}_{i} \mathrm{r}_{i}^{2}}-\frac{\mathfrak{m}_{i} \mathfrak{M}_{i}}{\mathrm{r}_{i}}\right) \\
& +\mu\left(\frac{y^{(1)} \cdot y^{(2)}}{m_{0}}-\frac{m_{1} m_{2}}{\left|x^{(1)}-x^{(2)}\right|}\right)(\mathrm{R}, \Phi, \mathrm{r}, \phi, ; \mathrm{G})
\end{aligned}
$$

denote the four degrees of freedom function which is obtained from $\mathrm{H}_{3 \mathrm{~b}}(\mathrm{R}, \Phi, \mathrm{H}, \mathrm{r}, \varphi, \mathrm{h})$ using relations (11), (32), (33) and (35) with G regarded as an external fixed parameter. Following Arnold, we might say that the Hamiltonian $\mathrm{H}_{3 \mathrm{~b} \text {,red }}(\mathrm{R}, \Phi, \mathrm{r}, \varphi ; \mathrm{G})$ "resembles the Hamiltonian of a certain planar problem ${ }^{13}$ ". Radau proved (with a computational procedure) that the motion of the eight coordinates $\left(\mathrm{R}_{i}, \Phi_{i}, \mathrm{r}_{i}, \varphi_{i}\right)_{1 \leq i \leq 2}$ is governed by the Hamilton equations of $\mathrm{H}_{3 \mathrm{~b} \text {, red }}(\mathrm{R}, \Phi, \mathrm{r}, \varphi ; \mathrm{G})$. This result may be formulated by saying that, for any fixed value of $\mathrm{G}$, the imbedding

$$
\left(\mathrm{R}_{1}, \mathrm{R}_{2}, \Phi_{1}, \Phi_{2}, \mathrm{r}_{1}, \mathrm{r}_{2}, \varphi_{1}, \varphi_{2}\right) \in \mathbb{R}^{8} \rightarrow\left(y^{(1)}, y^{(2)}, x^{(1)}, x^{(2)}\right) \in \mathbb{R}^{12}
$$

defined by Eqs. (11), (32), (33) and (35) is a canonical map for the eight-dimensional manifold (34).

Let us incidentally mention that Radau's result (that he proved in about sixty pages) can be obtained as a consequence of a more general and global result that has been found one century later by A. Deprit [10]. Deprit also extended it to the case of more planets, as described in the next section.

To conclude this section dedicated to Jacobi-Radau's procedure, we describe two "planetary" modifications of Jacobi-Radau variables, which are fitted to our needs.

Firstly, we switch from the variables $\left(\mathrm{R}_{i}, \Phi_{i}, \mathrm{r}_{i}, \varphi_{i}\right)$ to the variables on the left in (18). Then also the imbedding ${ }^{14}$

$$
\left(\Lambda_{1}, \Lambda_{2}, \Gamma_{1}, \Gamma_{2}, \ell_{1}, \ell_{2}, \mathrm{~g}_{1}, \mathrm{~g}_{2}\right) \in \mathbb{R}^{4} \times \mathbb{T}^{4} \rightarrow\left(y^{(1)}, y^{(2)}, x^{(1)}, x^{(2)}\right) \in \mathbb{R}^{12}
$$

is a canonical Kepler map, restricted to the same manifold (34). Such change reduces the twobody terms in (29) to their Keplerian form in (16).

\footnotetext{
${ }^{12}$ Note that, in principle, $\mathrm{G}$ is a function of $\Phi_{1}, \Phi_{2}, \mathrm{H}_{1}, \mathrm{H}_{2}$ and $\mathrm{h}_{1}-\mathrm{h}_{2}$.

${ }^{13}$ The verbatim citation is taken from $[2$, end of $\S 4$, p. 141$]$. Here the reference to the planar problem is just relatively to the number of degrees of freedom. Note however that the spatial and the planar three-body problems have the same number of degrees of freedom in the situation that the spatial problem is completely reduced and the planar one is not. The completely reduced planar problem has indeed three degrees of freedom, not four.

${ }^{14}$ The map (37) is attributed to Jacobi by Arnold, while the map (36) is attributed to Radau by A. Deprit: compare [2, beginning of $\S 4$, p.141] and [10, end of p. 187].
} 
Secondly, since the map in (18) is singular for zero eccentricities, it is customary to switch to a set of Poincaré-like coordinates

$$
\left(\Lambda_{1}, \Lambda_{2}, \hat{\lambda}_{1}, \hat{\lambda}_{2}, \hat{\eta}_{1}, \hat{\eta}_{2}, \hat{\xi}_{1}, \hat{\xi}_{2}\right)
$$

where the "secular variables" $\left(\Gamma_{1}, \Gamma_{2}, \mathrm{~g}_{1}, \mathrm{~g}_{2}\right)$ have been replaced by

$$
\hat{\eta}_{i}=\sqrt{2\left(\Lambda_{i}-\Gamma_{i}\right)} \cos g_{i} \quad \hat{\xi}_{i}=-\sqrt{2\left(\Lambda_{i}-\Gamma_{i}\right)} \sin g_{i}
$$

and the fast angles $\left(\ell_{1}, \ell_{2}\right)$ by

$$
\hat{\lambda}_{i}=\ell_{i}+g_{i}
$$

The change

$$
\left(\Lambda_{1}, \Lambda_{2}, \hat{\lambda}_{1}, \hat{\lambda}_{2}, \hat{\eta}_{1}, \hat{\eta}_{2}, \hat{\xi}_{1}, \hat{\xi}_{2}\right) \rightarrow\left(\Lambda_{1}, \Lambda_{2}, \Gamma_{1}, \Gamma_{2}, \ell_{1}, \ell_{2}, g_{1}, g_{2}\right)
$$

is canonical and induces an imbedding

$$
\left(\Lambda_{1}, \Lambda_{2}, \hat{\lambda}_{1}, \hat{\lambda}_{2}, \hat{\eta}_{1}, \hat{\eta}_{2}, \hat{\xi}_{1}, \hat{\xi}_{2}\right) \rightarrow\left(y^{(1)}, y^{(2)}, x^{(1)}, x^{(2)}\right) \in \mathbb{R}^{12}
$$

which regular ${ }^{15}$ for $\left(\hat{\mathfrak{\eta}}_{i}, \hat{\xi}_{i}\right)=0$. Recall that $\left(\hat{\mathfrak{\eta}}_{i}, \hat{\xi}_{i}\right)=0$ corresponds to the vanishing of the $i^{\text {th }}$ eccentricity.

For future reference, we just mention that the maps in (37) and (39) have the form, respectively

$$
\left\{\begin{array} { l } 
{ x ^ { ( 1 ) } = \mathrm { R } _ { 1 } ( \iota _ { 1 } ) \overline { x } _ { \mathrm { D } } ^ { ( 1 ) } ( \Lambda _ { 1 } , \Gamma _ { 1 } , \ell _ { 1 } , \mathrm { g } _ { 1 } ) } \\
{ x ^ { ( 2 ) } = \mathrm { R } _ { 1 } ( - \iota _ { 2 } ) \overline { x } _ { \mathrm { D } } ^ { ( 2 ) } ( \Lambda _ { 1 } , \Gamma _ { 1 } , \ell _ { 1 } , \mathrm { g } _ { 1 } ) }
\end{array} \quad \left\{\begin{array}{l}
y^{(1)}=\mathrm{R}_{1}\left(\iota_{1}\right) \bar{y}_{\mathrm{D}}^{(1)}\left(\Lambda_{2}, \Gamma_{2}, \ell_{2}, \mathrm{~g}_{2}\right) \\
y^{(2)}=\mathrm{R}_{1}\left(-\iota_{2}\right) \bar{y}_{\mathrm{D}}^{(2)}\left(\Lambda_{2}, \Gamma_{2}, \ell_{2}, \mathrm{~g}_{2}\right)
\end{array}\right.\right.
$$

and

$$
\left\{\begin{array} { l } 
{ x ^ { ( 1 ) } = \mathrm { R } _ { 1 } ( \iota _ { 1 } ) \overline { x } _ { \mathrm { P } } ^ { ( 1 ) } ( \Lambda _ { 1 } , \hat { \lambda } _ { 1 } , \hat { \eta } _ { 1 } , \hat { \xi } _ { 1 } ) } \\
{ x ^ { ( 2 ) } = \mathrm { R } _ { 1 } ( - \iota _ { 2 } ) \overline { x } _ { \mathrm { P } } ^ { ( 2 ) } ( \Lambda _ { 1 } , \hat { \lambda } _ { 1 } , \hat { \eta } _ { 1 } , \hat { \xi } _ { 1 } ) }
\end{array} \quad \left\{\begin{array}{l}
y^{(1)}=\mathrm{R}_{1}\left(\iota_{1}\right) \bar{y}_{\mathrm{P}}^{(1)}\left(\Lambda_{2}, \hat{\lambda}_{2}, \hat{\eta}_{2}, \hat{\xi}_{2}\right) \\
y^{(2)}=\mathrm{R}_{1}\left(-\iota_{2}\right) \bar{y}_{\mathrm{P}}^{(2)}\left(\Lambda_{2}, \hat{\lambda}_{2}, \hat{\eta}_{2}, \hat{\xi}_{2}\right)
\end{array}\right.\right.
$$

where $\left(\Lambda_{i}, \Gamma_{i}, \ell_{i}, \mathrm{~g}_{i}\right) \rightarrow\left(\bar{x}_{\mathrm{D}}^{(i)}, \bar{y}_{\mathrm{D}}^{(i)}\right),\left(\Lambda_{i}, \hat{\lambda}_{i}, \hat{\eta}_{i}, \hat{\xi}_{i}\right) \rightarrow\left(\bar{x}_{\mathrm{P}}^{(i)}, \bar{y}_{\mathrm{P}}^{(i)}\right)$ denote the planar Delaunay, Poincaré maps, and $\iota_{1}, \iota_{2}$ have suitable expressions in such sets of coordinates.

Moreover, we denote as

$$
\begin{aligned}
\mathrm{H}_{3 \mathrm{~b}, \text { red,plt }} & =-\sum_{1 \leq i \leq 2} \frac{\mathfrak{m}_{i}^{3} \mathfrak{M}_{i}^{2}}{2 \Lambda_{i}}+\mu\left(\frac{y^{(1)} \cdot y^{(2)}}{m_{0}}-\frac{m_{1} m_{2}}{\left|x^{(1)}-x^{(2)}\right|}\right)(\Lambda, \Gamma, \ell, \gamma ; \mathrm{G}) \\
\mathrm{H}_{3 \mathrm{~b}, \text { red,plt,reg }} & =-\sum_{1 \leq i \leq 2} \frac{\mathfrak{m}_{i}^{3} \mathfrak{M}_{i}^{2}}{2 \Lambda_{i}}+\mu\left(\frac{y^{(1)} \cdot y^{(2)}}{m_{0}}-\frac{m_{1} m_{2}}{\left|x^{(1)}-x^{(2)}\right|}\right)(\Lambda, \hat{\lambda}, \hat{\mathrm{z}} ; \mathrm{G})
\end{aligned}
$$

with $\hat{z}=\left(\hat{\eta}_{1}, \hat{\eta}_{2}, \hat{\xi}_{1}, \hat{\xi}_{2}\right)$, the three-body written in the latter two different sets of variables.

\subsection{Boigey-Deprit's reduction for $(1+n)$ bodies}

In 1893 A. Deprit [10], strongly influenced by a previous paper by F. Boigey ${ }^{16}$ [3] discovered a set of symplectic variables defined on the $6 n$-dimensional space phase that (suitably modified) "unfold"

\footnotetext{
${ }^{15}$ However, the imbedding (39) is singular when the mutual inclination vanishes. We should mention, at this respect, that, even though the change of coordinates (39) is singular for zero mutual inclination, the Hamiltonian

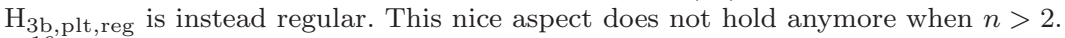

${ }_{16}$ The paper by Boigey deals with the case of four bodies $(n=3)$. Boigey obtains a slightly less general result than the one by Deprit, since she constructs coordinates on a suitable manifold, not on the whole phase space. Boigey's coordinates may be obtained as Deprit's coordinates for four bodies restricted to Boigey's manifold.
} 
Jacobi-Radau's reduction of the nodes described in the previous section. Deprit's variables are defined in general for any many-particle system

$$
\mathrm{H}\left(y^{(1)}, \cdots, y^{(n)}, x^{(1)}, \cdots, y^{(n)}\right)
$$

with general $n \geq 2$, which has the integral $\mathrm{C}$ in (8) preserved. They are not specialized to the planetary problem, but may be adapted to it, as described in the next $\S 4.3$. Deprit's variables remained unnoticed for many years apart for some applications to the three-body problem in which case, as said, they trivialize to Jacobi-Radau's reduction, described in $§ 4.1$. See, for example, the paper [14], where the Hamiltonian is just the same as in [15], [20], which are previous to Deprit's paper. Deprit's reduction has been next rediscovered (in the form given by the map (53); see also [6]) by the author during her $\mathrm{PhD}$, under the motivation of their application to the direct proof of Arnold's Theorem.

Analogously to the case of two planets, also Deprit's construction requires that certain inclinations appearing in the construction do not vanish. Let us start with the case $n=2$, in order to compare with Jacobi-Radau's procedure.

Let $\mathrm{R}_{1}, \mathrm{R}_{2}, \mathrm{r}_{1}, \mathrm{r}_{2}, \Phi_{1}$ and $\Phi_{2}$ be as in (10). Let

$$
\nu_{1}:=\mathrm{C} \times \mathrm{C}^{(1)} \quad \nu_{2}:=\mathrm{C} \times \mathrm{C}^{(2)} .
$$

Clearly, $\nu_{1}$ and $\nu_{2}$ are opposite, since

$$
\nu_{1}+\nu_{2}=\mathrm{C} \times \mathrm{C}^{(1)}+\mathrm{C} \times \mathrm{C}^{(2)}=\mathrm{C} \times \mathrm{C}=0 .
$$

This relation is an extension of (30). Define

$$
\nu_{0}:=k^{(3)} \times \mathrm{C} .
$$

Then let

$$
\mathrm{C}_{3}:=\mathrm{C} \cdot k^{(3)} \quad \mathrm{G}:=|\mathrm{C}| \quad \zeta:=\alpha_{k^{(3)}}\left(k^{(1)}, \nu_{0}\right) \quad \mathrm{g}:=\alpha_{\mathrm{C}}\left(\nu_{0}, \nu_{1}\right)
$$

and

$$
\phi_{1}:=\alpha_{\mathrm{C}^{(1)}}\left(\nu_{1}, x^{(1)}\right) \quad \phi_{2}:=\alpha_{\mathrm{C}^{(2)}}\left(\nu_{2}, x^{(2)}\right)=\alpha_{\mathrm{C}^{(2)}}\left(\nu_{1}, x^{(2)}\right)+\pi .
$$

Deprit proved that the twelve-dimensional change

$$
\left(\mathrm{C}_{3}, \mathrm{G}, \mathrm{R}_{1}, \mathrm{R}_{2}, \Phi_{1}, \Phi_{2}, \zeta, \mathrm{g}, \mathrm{r}_{1}, \mathrm{r}_{2}, \phi_{1}, \phi_{2}\right) \rightarrow\left(y^{(1)}, y^{(2)}, x^{(1)}, x^{(2)}\right)
$$

is homogeneous.

The main point (which holds also for the case $n \geq 3$ described below) is that $\mathrm{C}_{3}, \zeta$ and g are cyclic in any $\mathrm{SO}(3)$-invariant Hamiltonian, since they are conjugated to integrals. The existence of two cyclic conjugated variables is just the cause of the degeneracy of all the orders of Birkhoff normal form for the secular perturbing function written in Poincaré variables, found in [7]. The fact that an action, $\mathrm{C}_{3}$, is cyclic is a remarkable situation, which generalizes what we already know from the two-body Hamiltonian (16) (compare §3.1). We remark, at this respect that Deprit seemed not to notice this fact, since, at the end of $\S 4$, he underlines the cyclicality of $\zeta$ and g but not the one of $\mathrm{C}_{3}$.

Let us inspect the analytical expression of the map (45). By the definitions, if $\iota_{0}$ is the convex angle formed by $\mathrm{C}$ and $k^{(3)}$, determined by

$$
\cos \iota_{0}=\frac{\mathrm{C}_{3}}{\mathrm{G}}
$$


then the map (45) is given by

$$
\begin{aligned}
& \left\{\begin{array}{l}
x^{(1)}=\mathrm{R}^{(3)}(\zeta) \mathrm{R}^{(1)}\left(\iota_{0}\right) \mathrm{R}^{(3)}(\mathrm{g}) \mathrm{R}^{(1)}\left(\iota_{1}\right) \bar{x}^{(1)} \\
x^{(2)}=\mathrm{R}^{(3)}(\zeta) \mathrm{R}^{(1)}\left(\iota_{0}\right) \mathrm{R}^{(3)}(\mathrm{g}+\pi) \mathrm{R}^{(1)}\left(\iota_{2}\right) \bar{x}^{(2)}
\end{array}\right. \\
& \left\{\begin{array}{l}
y^{(1)}=\mathrm{R}^{(3)}(\zeta) \mathrm{R}^{(1)}\left(\iota_{0}\right) \mathrm{R}^{(3)}(\mathrm{g}) \mathrm{R}^{(1)}\left(\iota_{1}\right) \bar{y}^{(1)} \\
y^{(2)}=\mathrm{R}^{(3)}(\zeta) \mathrm{R}^{(1)}\left(\iota_{0}\right) \mathrm{R}^{(3)}(\mathrm{g}+\pi) \mathrm{R}^{(1)}\left(\iota_{2}\right) \bar{y}^{(2)}
\end{array}\right.
\end{aligned}
$$

with $\bar{x}^{(1)}, \bar{x}^{(2)}, \bar{y}^{(1)}, \bar{y}^{(2)}$ as in (11) with $\varphi_{i}$ replaced by $\phi_{i}$. The product $\mathrm{R}^{(3)}(\zeta) \mathrm{R}^{(1)}\left(\iota_{0}\right) \mathrm{R}^{(3)}(\mathrm{g})$ which is in common in all such formulae is negligible by invariance by rotations. Therefore, in view of expressing the three-body Hamiltonian (29) in terms of Deprit variables (at l. h. s. of (45)), the angles $\zeta, \iota_{0}$ and g can be fixed to any arbitrary value. Fixing them all to zero reduces (46) to (32)-(33) with $\varphi_{i}$ replaced by $\phi_{i}$. In particular, Radau's theorem mentioned in $\S 4.1$ is clarified $^{17}$ from the geometrical point of view. Note in fact that the manifold (34) in terms of the variables on the left in (45) has the simple expression

$$
\mathrm{C}_{3}=\mathrm{G}_{0} \quad \mathrm{G}=\mathrm{G}_{0} \quad \zeta=0 \quad \mathrm{~g}=0 .
$$

Let us now describe how Deprit extended his reduction to the case of more than two planets.

Let $\mathrm{R}_{i}, \Phi_{i}, \mathrm{r}_{i}$ as in (10), with $1 \leq i \leq n$. For $1 \leq j \leq n$ define

$$
\mathrm{C}^{(j)}:=x^{(j)} \times y^{(j)} \quad \mathrm{S}^{(j)}:=\sum_{j \leq i \leq n} \mathrm{C}^{(i)}
$$

so that $\mathrm{S}^{(1)}=\mathrm{C}, \mathrm{S}^{(n)}=\mathrm{C}^{(n)}$. Then let $\nu_{0}$ as in (43) and, for $1 \leq j \leq n-1$,

$$
\nu_{j}:=\mathrm{S}^{(j)} \times \mathrm{C}^{(j)} \quad 1 \leq j \leq n-1 ; \quad \nu_{n}:=\mathrm{S}^{(n-1)} \times \mathrm{C}^{(n)} .
$$

There are just $n$ independent $\nu_{j}$ 's (with $0 \leq j \leq n-1$ ), since $\nu_{n-1}$ and $\nu_{n}$ are opposite. Assume that none of the $\nu_{j}$ 's vanishes. Then define $\mathrm{C}_{3}, \zeta$ as in $(44)$ and $^{18}$

$$
\left\{\begin{array}{l}
\Psi_{j-1}:=\left|\mathrm{S}^{(j)}\right| \\
\psi_{j-1}:=\alpha_{\mathrm{S}^{(j)}}\left(\nu_{j-1}, \nu_{j}\right)
\end{array} \quad \phi_{i}:=\alpha_{\mathrm{C}^{(i)}}\left(\nu_{i}, x^{(i)}\right)\right.
$$

with $1 \leq i \leq n, 1 \leq j \leq n-1$. Then the variables $\Psi_{0}:=\mathrm{G}$ and $\psi_{0}:=\mathrm{g}$ are as in (44), with $\mathrm{C}$ the total angular momentum of $n$ planets. Define, finally,

$$
\begin{aligned}
& \mathrm{R}:=\left(\mathrm{R}_{1}, \cdots, \mathrm{R}_{n}\right) \quad \mathrm{r}:=\left(\mathrm{r}_{1}, \cdots, \mathrm{r}_{n}\right) \quad \Phi:=\left(\Phi_{1}, \cdots, \Phi_{n}\right) \\
& \Psi:=\left(\mathrm{C}_{3}, \Psi_{0}, \cdots, \Psi_{n-2}\right) \quad \phi:=\left(\phi_{1}, \cdots, \phi_{n}\right) \quad \psi:=\left(\zeta, \psi_{0}, \cdots, \psi_{n-2}\right) .
\end{aligned}
$$

Deprit proved that the change

$$
(\mathrm{R}, \Phi, \Psi, \mathrm{r}, \phi, \psi) \in \mathbb{R}^{3 n} \times \mathbb{T}^{3 n} \rightarrow\left(y^{(1)}, \cdots, y^{(n)}, x^{(1)}, \cdots, x^{(n)}\right) \in \mathbb{R}^{3 n} \times \mathbb{R}^{3 n}
$$

\footnotetext{
${ }^{17}$ In [10, end of p. 194] we read "The intention [of this note] was to show how the global symmetry with respect to the group $\mathrm{SO}(3)$... affords a suitable coordinate system leading without artificiality the reduction of the nodes."

${ }^{18}$ Here we use notations and definitions closer to Deprit's. In [8] a different, but equivalent, definition of the variables $(\Psi, \psi)$ has been used. In $[10]\left(\mathrm{C}_{3}, \zeta\right)$ are called $\left(\mathrm{N}_{0}^{*}, \nu_{0}^{*}\right)$, while $\Psi_{0}, \cdots, \Psi_{n-2}, \psi_{0}, \cdots, \psi_{n-2}$ are called $\Theta_{0}^{*}, \cdots, \Theta_{n-2}^{*}, \theta_{0}^{*}, \cdots, \theta_{n-2}^{*}$. Moreover $\Phi_{1}, \cdots, \Phi_{n}, \phi_{1}, \cdots, \phi_{n}$ are called $\Theta_{1}, \cdots, \Theta_{n}, \theta_{1}, \cdots, \theta_{n}$ in [10]. With respect to the notations used in [8], the correspondence is the following. Calling here $\left(\Lambda^{*}, \Gamma^{*}, \Psi^{*}, \ell^{*}, \gamma^{*}, \psi^{*}\right)$ the coordinates that in [8] are named $(\Lambda, \Gamma, \Psi, \ell, \gamma, \psi)$, then $\left(\Lambda_{i}, \Gamma_{i}, \ell_{i}, \gamma_{i}\right)=\left(\Lambda_{n-i+1}^{*}, \Gamma_{n-i+1}^{*}, \ell_{n-i+1}^{*}, \gamma_{n-i+1}^{*}\right)$, and $\left(\Psi_{j}, \psi_{j}\right):=\left(\Psi_{n-j-1}^{*}, \psi_{n-j-1}\right.$, with $1 \leq i \leq n,-1 \leq j \leq n-1,\left(\Psi_{-1}, \psi_{-1}\right)$ as in $(55)$.
} 
is homogeneous.

The relative formulae to obtain any of the couples $\left(y^{(i)}, x^{(i)}\right)$ are a bit involved. They however can be found following suitable ${ }^{19}$ chains of frames

$$
\mathrm{F}_{0} \rightarrow \mathrm{F}_{1}^{*} \rightarrow \cdots \rightarrow \mathrm{F}_{i}^{*} \rightarrow \mathrm{F}_{i} \quad 1 \leq i \leq n,
$$

where $\mathrm{F}_{0}$ is a prefixed frame, $\mathrm{F}_{1}^{*}, \cdots, \mathrm{F}_{i}^{*}$ are auxiliary frames having their third axes in the direction of $\mathrm{S}^{(1)}, \cdots, \mathrm{S}^{(i)}$, respectively, $\mathrm{F}^{(i)}$ is the $i^{\text {th }}$ orbital frame, having its third axis in the direct of $\mathrm{C}^{(i)}$.

Easy geometric arguments lead, for the map (45), to expressions of the form

$$
\begin{aligned}
& x^{(i)}= \begin{cases}\mathcal{R}\left(\zeta, \iota_{0}\right) \mathcal{R}\left(\psi_{0}, \iota_{1}\right) \bar{x}^{(1)} & i=1 \\
\mathcal{R}\left(\zeta, \iota_{0}\right) \mathcal{R}\left(\psi_{0}+\pi, \iota_{1}^{*}\right) \cdots \mathcal{R}\left(\psi_{i-2}+\pi, \iota_{i}^{*}\right) \mathcal{R}\left(\psi_{i-1}, \iota_{i}\right) \bar{x}^{(i)} & 2 \leq i \leq n-1 \\
\mathcal{R}\left(\zeta, \iota_{0}\right) \mathcal{R}\left(\psi_{0}+\pi, \iota_{1}^{*}\right) \cdots \mathcal{R}\left(\psi_{n-2}+\pi, \iota_{n}^{*}\right) \bar{x}^{(n)} & i=n\end{cases} \\
& y^{(i)}= \begin{cases}\mathcal{R}\left(\zeta, \iota_{0}\right) \mathcal{R}\left(\psi_{0}, \iota_{1}\right) \bar{y}^{(1)} & i=1 \\
\mathcal{R}\left(\zeta, \iota_{0}\right) \mathcal{R}\left(\psi_{0}+\pi, \iota_{1}^{*}\right) \cdots \mathcal{R}\left(\psi_{i-2}+\pi, \iota_{i}^{*}\right) \mathcal{R}\left(\psi_{i-1}, \iota_{i}\right) \bar{y}^{(i)} & 2 \leq i \leq n-1 \\
\mathcal{R}\left(\zeta, \iota_{0}\right) \mathcal{R}\left(\psi_{0}+\pi, \iota_{1}^{*}\right) \cdots \mathcal{R}\left(\psi_{n-2}+\pi, \iota_{n}\right) \bar{y}^{(n)} & i=n\end{cases}
\end{aligned}
$$

where $\bar{x}^{(i)}, \bar{y}^{(i)}$ depend on $\left(\mathrm{R}_{i}, \Phi_{i}, \mathrm{r}_{i}, \phi_{i}\right)$ as in $(11)$, with $\varphi_{i}$ replaced by $\phi_{i} ; \mathcal{R}(\psi, \iota):=\mathrm{R}^{(3)}(\psi) \mathrm{R}^{(1)}(\iota)$; $\iota_{i}$ are the convex angles formed by $\mathrm{S}^{(i)}$ and $\mathrm{C}^{(i)}$, while $\iota_{j}^{*}$ are the convex angles formed by $\mathrm{S}^{(j)}$ and $\mathrm{S}^{(j+1)}$. Such convex angles are determined by

$$
\cos \iota_{i}=\frac{\Psi_{i-1}^{2}+\Phi_{i}^{2}-\Psi_{i}^{2}}{2 \Psi_{i-1} \Phi_{i}} \quad \cos \iota_{j}^{*}=\frac{\Psi_{j}^{2}+\Phi_{j}^{2}-\Psi_{j-1}^{2}}{2 \Psi_{j} \Phi_{j}}
$$

with $1 \leq i \leq n$ and $1 \leq j \leq n-1$.

The description given in the formulae above (developed in [23]; see also [8, Appendix A]) is different from Deprit's approach, who preferred to use quaternions and, at the end, did not provide complete formulae ${ }^{20}$, since they seemed too complicate to him. We should add, at this respect, that Deprit believed that his variables would never ${ }^{21}$ be useful. His variables (in the rediscovered planetary version; compare the next section) have been instead the starting point for the proof of existence and non-degeneracy of the Birkhoff normal form for the planetary system and hence the complete, constructive proof of Arnold's Theorem [23], [8].

From the formulae (50) we have an explicit explanation of the reduction: for $\mathrm{SO}(3)$-invariant systems, the three first rotations by the angles $\zeta, \iota_{0}$ and $\psi_{0}$ in front of any $x^{(i)}$ of $y^{(i)}$ may be neglected and, as expected, only the variables

$$
(\mathrm{R}, \Phi, \hat{\Psi}, \mathrm{r}, \phi, \hat{\psi})
$$

\footnotetext{
${ }^{19}$ Here, $\mathrm{F}_{0}$ is the prefixed frame, $\mathrm{F}_{1}^{*}$ has the first axis in the direction of $\nu_{0}$ and the third one in the direction of $\mathrm{S}^{(1)} ; \mathrm{F}_{n}:=\mathrm{F}_{n}^{*}$, where $\mathrm{F}_{j}^{*}$, for $2 \leq j \leq i \leq n$ has the first axis in the direction of $\mathrm{S}^{(j-1)} \times \mathrm{S}^{(j)}=-\nu_{j-1}$ and the third one in the direction of $\mathrm{S}^{(j)} ; \mathrm{F}_{i}$, for $1 \leq i \leq n-1$, has the first axis in the direction of $\nu_{i}=\mathrm{S}^{(i-1)} \times \mathrm{C}^{(j)}=\nu_{i}$ and the third axis in the direction of $\mathrm{C}^{(i)}$.

${ }^{20}$ In [10, end of $\left.\S 4\right]$ we read "The final expressions increase in complexity; it serves no purpose to enter the results in this Note."

${ }^{21}$ At the end of p. 194 of Deprit's paper, we find "Whether the new phase variables are practical in the General Theory of Perturbation is an open question. At least, for planetary theories, the answer is likely to be negative: the tree of kinetic frames imposes a recursive hierarchy without physical correspondence in the solar system."
} 
where

$$
\hat{\Psi}:=\left(\Psi_{1}, \cdots, \Psi_{n-2}\right) \quad \hat{\psi}:=\left(\psi_{1}, \cdots, \psi_{n-2}\right)
$$

will appear and the integral/action $\Psi_{0}=\mathrm{G}$ will play the rôle of an external parameter.

For example, choosing the planetary heliocentric Hamiltonian $\mathrm{H}_{\text {hel }}$ in (2), one finds

$$
\begin{aligned}
\mathrm{H}_{\text {Dep }} & =\sum_{1 \leq i \leq n}\left(\frac{\mathrm{R}_{i}^{2}}{2 \mathfrak{m}_{i}}+\frac{\Phi_{i}^{2}}{2 \mathfrak{m}_{i} \mathrm{r}_{i}^{2}}-\frac{\mathfrak{m}_{i} \mathfrak{M}_{i}}{\mathrm{r}_{i}}\right) \\
& +\mu \sum_{1 \leq i<j \leq n}\left(\frac{y^{(i)} \cdot y^{(j)}}{m_{0}}-\frac{m_{i} m_{j}}{\left|x^{(i)}-x^{(j)}\right|}\right)(\mathrm{R}, \Phi, \hat{\Psi}, \mathrm{r}, \phi, \hat{\psi} ; \mathrm{G}) .
\end{aligned}
$$

\subsection{Planetary version of Deprit's variables and regularizations}

The planetary version of Deprit's coordinates (which is not mentioned in [10]) may obtained via the change

$$
\left(\Lambda_{i}, \Gamma_{i}, \ell_{i}, \gamma_{i}\right) \rightarrow\left(\mathrm{R}_{i}, \Phi_{i}, \mathrm{r}_{i}, \phi_{i}\right)
$$

defined via the integration of the two-body terms (14) terms appearing in (51). With this change we then have a symplectic map

$$
(\Lambda, \Gamma, \Psi, \ell, \gamma, \psi) \rightarrow\left(y^{(1)}, \cdots, y^{(n)}, x^{(1)}, \cdots, x^{(n)}\right),
$$

which turns out to be a Kepler map in the sense of $\S 3.2$. More precisely, relations (24)-(25) are implied by the fact that the planar Delaunay map is used.

The Hamiltonian $\mathrm{H}_{\text {Dep }}$ takes the "planetary" form

$$
\mathrm{H}_{\text {Dep }, \mathrm{plt}}=-\sum_{1 \leq i \leq n} \frac{\mathfrak{m}_{i}^{3} \mathfrak{M}_{i}^{2}}{2 \Lambda_{i}}+\mu \sum_{1 \leq i<j \leq n}\left(\frac{y^{(i)} \cdot y^{(j)}}{m_{0}}-\frac{m_{i} m_{j}}{\left|x^{(i)}-x^{(j)}\right|}\right)(\Lambda, \Gamma, \hat{\Psi}, \ell, \gamma, \hat{\psi} ; \mathrm{G})
$$

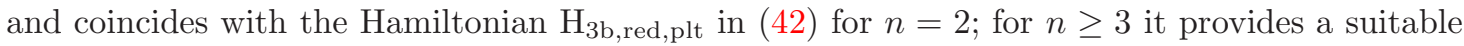
extension to it. It can be used for planetary theories in the case eccentricities and inclinations are not required to be small. Indeed, such occurrences are singular for $\mathrm{H}_{\mathrm{Dep}, \mathrm{plt}}$ : the singularities of inclinations is due to the definition of the variables $(\hat{\Psi}, \hat{\psi})$ (compare $\S 4.2)$; the singularities for eccentricities are introduced by the maps (52). For the reader who is interested (for application purposes) to the analytical expression of the map (53), we just mention that such map is completely analogous to the map in (50), apart for taking $\Phi_{i}=\Gamma_{i}$ and replacing $\bar{x}^{(i)}\left(\mathrm{R}_{i}, \Phi_{i}, \mathrm{r}_{i}, \phi_{i}\right)$, $\bar{y}^{(i)}\left(\mathrm{R}_{i}, \Phi_{i}, \mathrm{r}_{i}, \phi_{i}\right)$, with $\bar{x}_{\mathrm{D}}^{(i)}\left(\Lambda_{i}, \Gamma_{i}, \ell_{i}, \gamma_{i}\right), \bar{y}_{\mathrm{D}}^{(i)}\left(\Lambda_{i}, \Gamma_{i}, \ell_{i}, \gamma_{i}\right)$, where the maps

$$
\bar{\phi}_{\mathrm{D}}^{(i)}: \quad\left(\Lambda_{i}, \Gamma_{i}, \ell_{i}, \gamma_{i}\right) \rightarrow\left(\bar{y}_{\mathrm{D}}^{(i)}\left(\Lambda_{i}, \Gamma_{i}, \ell_{i}, \gamma_{i}\right), \bar{x}_{\mathrm{D}}^{(i)}\left(\Lambda_{i}, \Gamma_{i}, \ell_{i}, \gamma_{i}\right)\right)
$$

are as in (20). For $n=2$, it reduces to (40) neglecting the three first cyclic rotations by the angles $\zeta, \iota_{0}, \psi_{0}$.

To deal with the case when eccentricities or inclinations may also vanish, it is possible to switch to new sets of coordinates, analogously to Poincaré's procedure for regularizing Delaunay coordinates. Unfortunately, it is not possible to regularize all singularities and, simultaneously, keep the number of degrees of freedom to $(3 n-2)$. This may be compared with the situation for the three-body case (see $\S 4.1$ ), where, as mentioned, the coordinates (38) are suited for a four degrees of freedom system, but the configuration with zero mutual inclination is singular.

We may then choose if regularizing all of vanishing eccentricities or inclinations, at the cost of enhancing the number of degrees of freedom by one ("partial reduction"), or, alternatively, all but one ("full reduction"). Moreover (see §4.4) full reduction, in general, breaks down the natural symmetries of the problem; compare also [21]. 


\subsubsection{Partial reduction (RPS variables)}

The complete regularization is obtained replacing the map (53) with a map

$$
(\Lambda, \lambda, z) \in \mathbb{R}^{n} \times \mathbb{T}^{n} \times \mathbb{R}^{4 n} \rightarrow\left(y^{(1)}, \cdots, y^{(n)}, x^{(1)}, \cdots, x^{(n)}\right)
$$

where

$$
z=\left(\eta_{1}, \cdots, \eta_{n}, \xi_{1}, \cdots, \xi_{n}, p_{0}, \cdots, p_{n-1}, q_{0}, \cdots, q_{n-1}\right)
$$

which is regular for $\left(\eta_{i}, \xi_{i}\right),\left(p_{j}, q_{j}\right)$ approaching $(0,0)(1 \leq i \leq n, 0 \leq j \leq n-1)$ and such that $\left(\eta_{i}, \xi_{i}\right)=0$ corresponds to the vanishing of the $i^{\text {th }}$ eccentricity, $\left(p_{j}, q_{j}\right)=0$ corresponds to the vanishing of the node $\nu_{j}$ defined in $\S 4.2$. The explicit formulae of the map (54) are given in [23], [8]. The variables $(\Lambda, \lambda, z)$ have been found in [23] and have been named "RPS" (Regular, Planetary and Symplectic) in [8]. Their complete definition is

$$
\begin{aligned}
& \lambda_{j}=\ell_{i}+\gamma_{i}+\psi^{(i-1)}, \quad \text { where } \quad \psi^{(j)}=\sum_{-1 \leq k \leq j} \psi_{k}, \\
& \left\{\begin{array}{l}
\eta_{i}=\sqrt{2\left(\Lambda_{i}-\Gamma_{i}\right)} \cos \left(\gamma_{i}+\psi^{(i-1)}\right) \\
\xi_{i}=-\sqrt{2\left(\Lambda_{i}-\Gamma_{i}\right)} \sin \left(\gamma_{i}+\psi^{(i-1)}\right)
\end{array}\right. \\
& \left\{\begin{array}{l}
p_{j}=\sqrt{2\left(\Gamma_{j}+\Psi_{j}-\Psi_{j-1}\right)} \cos \psi^{(j-1)} \\
q_{j}=-\sqrt{2\left(\Gamma_{j+1}+\Psi_{j+1}-\Psi_{j}\right)} \sin \psi^{(j-1)}
\end{array}\right.
\end{aligned}
$$

with

$$
\psi_{-1}:=\zeta, \Psi_{-1}:=\mathrm{C}_{3}, \Gamma_{0}:=0,1 \leq i \leq n, 0 \leq j \leq n-1 .
$$

Main points are

- The couple ${ }^{22}\left(p_{0}, q_{0}\right)$ is cyclic and plays the rôle of $\left(\mathrm{C}_{3}, \zeta\right)$ in the set on the left in $(53)$. Indeed,

$$
p_{0}=\sqrt{2\left(\mathrm{G}-\mathrm{C}_{3}\right)} \cos \zeta \quad q_{0}=-\sqrt{2\left(\mathrm{G}-\mathrm{C}_{3}\right)} \sin \zeta
$$

are both integrals;

- The variables $(\Lambda, \lambda, z)$ behave exactly as the Poincaré variables $(\Lambda, \lambda, z)$ of $\S 3.1$ for what concerns D'Alembert rules ${ }^{23}$. In particular, $\bar{z}=0$, where

$$
\bar{z}:=(\eta, \xi, \bar{p}, \bar{q}):=\left(\eta_{1}, \cdots, \eta_{n}, \xi_{1}, \cdots, \xi_{n}, p_{1}, \cdots, p_{n-1}, q_{1}, \cdots, q_{n-1}\right)
$$

is $z$ deprived of $\left(p_{0}, q_{0}\right)$, is an elliptic equilibrium point for the secular system associated to $\mathrm{H}_{\mathrm{rps}}$, where $\mathrm{H}_{\mathrm{rps}}(\Lambda, \lambda, \bar{z})$ is $\mathrm{H}_{\text {hel }}$ in (2) expressed in the variables $(\Lambda, \lambda, z)$. More details are in [23], [8], [7].

- The formulae relating the change of coordinates (54) have the form

$$
x^{(i)}=\left\{\begin{array}{ll}
\mathfrak{R}_{0}^{*} \mathfrak{R}_{1} \bar{x}_{\mathrm{P}}^{(1)} & i=1 \\
\mathfrak{R}_{0}^{*} \ldots \mathfrak{R}_{i}^{*} \mathfrak{R}_{i} \bar{x}_{\mathrm{P}}^{(i)} & 2 \leq i \leq n
\end{array} \quad y^{(i)}= \begin{cases}\mathfrak{R}_{0}^{*} \mathfrak{R}_{1} \bar{y}_{\mathrm{P}}^{(1)} & i=1 \\
\mathfrak{R}_{0}^{*} \ldots \mathfrak{R}_{i}^{*} \mathfrak{R}_{i} \bar{y}_{\mathrm{P}}^{(i)} & 2 \leq i \leq n\end{cases}\right.
$$

\footnotetext{
${ }^{22}$ Beware that here we are using different notations with respect to [8]. Letting $\Lambda^{*}, \lambda^{*} \eta^{*}, \xi^{*}, p^{*}, q^{*}$ the variables named $\Lambda, \lambda \eta, \xi, p, q$ in [8], the correspondence is: $\left(\Lambda_{i}, \lambda_{i}, \eta_{i}, \xi_{i}\right)=\left(\Lambda_{n-i+1}^{*}, \lambda_{n-i+1}^{*}, \eta_{n-i+1}^{*}, \xi_{n-i+1}^{*}\right) ;\left(p_{j}, q_{j}\right)=$ $\left(p_{n-j}^{*}, q_{n-j}^{*}\right)$, with $1 \leq i \leq n, 0 \leq j \leq n-1$.

${ }_{23}^{23}$ 'Alembert rules are the expressions of rotation and reflection transformations in terms of Poincaré variables. See, e.g. , [8], [7], [25] for notices.
} 
where $\mathfrak{R}_{n}^{*}=$ id, $\mathfrak{R}_{0}^{*}, \cdots, \mathfrak{R}_{i}^{*}, \mathfrak{R}_{i}$ are regular, unitary $3 \times 3$ matrices depending only on $(\Lambda, z)$; $\mathfrak{R}_{0}^{*}$ is completely negligible, since reduces to the identity for $\left(p_{0}, q_{0}\right)=0$; the remaining matrices $\mathfrak{R}_{1}^{*}, \cdots, \mathfrak{R}_{i}^{*}, \mathfrak{R}_{i}$ depend only on $(\Lambda, \bar{z})$ and reduce to the identity for $(\bar{p}, \bar{q})=0$. In particular, as well as the spatial Poincaré maps (22), also the map (54) reduces to the planar Poincaré maps (23) for $(p, q)=0$.

- Even in the $n=2$ case, the map (54) is qualitatively different from the map (41) obtained via Jacobi reduction of the nodes. Indeed, the map (54) is regular for all vanishing eccentricities and inclinations and reduces the system to five d.o.f., while the map (41) is regular for all vanishing eccentricities but singular for vanishing mutual inclination and reduces the system to four d.o.f. The natural extension of the map (41) to the case $n \geq 3$ is described in the following section.

\subsubsection{Full reduction}

Another full reduction (besides the one described in $\S 4.2$ ) is obtained giving up the regularization of the singular manifold $\left\{\nu_{1}=0\right\}$ (where $\nu_{1}$ is defined in (48)), corresponding of the parallelism of the three vectors $\mathrm{C}, \mathrm{C}^{(1)}$ and $\mathrm{S}^{(2)}$. The remaining eccentricities and inclinations may be described instead with regularity. The map realizing this reduction, restricted to the rotating manifold (34), has the form of an imbedding

$$
(\Lambda, \hat{\lambda}, \hat{z}) \in \mathbb{R}^{n} \times \mathbb{T}^{n} \times \mathbb{R}^{4 n-4} \rightarrow\left(y^{(1)}, \cdots, y^{(n)}, x^{(1)}, \cdots, x^{(n)}\right)
$$

with

$$
\begin{aligned}
& x^{(i)}= \begin{cases}\mathrm{R}^{(1)}\left(\iota_{1}\right) \bar{x}_{\mathrm{P}}^{(1)} & i=1 \\
\mathrm{R}^{(1)}\left(-\iota_{1}^{*}\right) \Re_{2} \cdots \Re_{i}^{*} \Re_{i} \bar{x}_{\mathrm{P}}^{(i)} & 2 \leq i \leq n\end{cases} \\
& y^{(i)}= \begin{cases}\mathrm{R}^{(1)}\left(\iota_{1}\right) \bar{y}_{\mathrm{P}}^{(1)} & i=1 \\
\mathrm{R}^{(1)}\left(-\iota_{1}^{*}\right) \Re_{2} \ldots \mathfrak{R}_{i}^{*} \Re_{i} \bar{y}_{\mathrm{P}}^{(i)} & 2 \leq i \leq n\end{cases}
\end{aligned}
$$

with $\psi_{0}^{*}:=\psi_{0}+\zeta$ and $\mathfrak{R}_{i}^{*}, \mathfrak{R}_{j}, \bar{x}_{\mathrm{P}}^{(j)}, \bar{y}_{\mathrm{P}}^{(j)}$ as in the previous section. The formulae (56) naturally extend the formulae for the $n=2$ case in (41).

We shall denote as

$$
\mathrm{H}_{\mathrm{rps}}=-\sum_{1 \leq i \leq n} \frac{\mathfrak{m}_{i}^{3} \mathfrak{M}_{i}^{2}}{2 \Lambda_{i}}+\mu \sum_{1 \leq i<j \leq n}\left(\frac{y^{(i)} \cdot y^{(j)}}{m_{0}}-\frac{m_{i} m_{j}}{\left|x^{(i)}-x^{(j)}\right|}\right)(\Lambda, \lambda, \bar{z})
$$

and

$$
\mathrm{H}_{\text {full red }}=-\sum_{1 \leq i \leq n} \frac{\mathfrak{m}_{i}^{3} \mathfrak{M}_{i}^{2}}{2 \Lambda_{i}}+\mu \sum_{1 \leq i<j \leq n}\left(\frac{y^{(i)} \cdot y^{(j)}}{m_{0}}-\frac{m_{i} m_{j}}{\left|x^{(i)}-x^{(j)}\right|}\right)(\Lambda, \hat{\lambda}, \hat{z} ; \mathrm{G})
$$

the planetary system, written in the variables described in $\S 4.3 .1-\S 4.3 .2$ respectively.

\subsection{Full reduction and reflection symmetries}

Reducing completely all the integrals implies that symmetries related to them will be eliminated from the Hamiltonian.

A natural question is whether it is possible to reduce the system by the integrals and keep, on the other side, parities due to those symmetries which are not related to integrals; like, for example, the transformations (1). 
To begin with, let us investigate what happens to reflection symmetries when the system is completely reduced by rotations according to the reductions for $n=2, n \geq 3$, described, respectively, in $\S 4.1$ or $\S 4.3 .2$.

In the three-body case we have that the system retains a suitable parity in the variables $\hat{z}=(\hat{\eta}, \hat{\xi})$, due to reflections. This can be seen analyzing the the maps (41): the changes

$$
\begin{aligned}
& \left(\Lambda_{i}, \hat{\lambda}_{i}, \hat{\eta}_{i}, \hat{\xi}_{i}\right) \rightarrow\left(\Lambda_{i},-\hat{\lambda}_{i},-\hat{\mathfrak{\eta}}_{i}, \hat{\xi}_{i}\right) \\
& \left(\Lambda_{i}, \hat{\lambda}_{i}, \hat{\eta}_{i}, \hat{\xi}_{i}\right) \rightarrow\left(\Lambda_{i}, \pi-\hat{\lambda}_{i}, \hat{\eta}_{i},-\hat{\xi}_{i}\right)
\end{aligned}
$$

correspond, respectively, to transform the projections $\left(\bar{y}_{\mathrm{P}}^{(i)}, \bar{x}_{\mathrm{P}}^{(i)}\right)=\left(\left(\bar{y}_{\mathrm{P}, 1}^{(i)}, \bar{y}_{\mathrm{P}, 2}^{(i)}\right),\left(\bar{x}_{\mathrm{P}, 1}^{(1)}, \bar{x}_{\mathrm{P}, 2}^{(i)}\right)\right)$ of the planar Delaunay map as follows:

$$
\begin{aligned}
& \left(\bar{y}_{\mathrm{P}, 1}^{(i)}, \bar{y}_{\mathrm{P}, 2}^{(i)}\right),\left(\bar{x}_{\mathrm{P}, 1}^{(i)}, \bar{x}_{\mathrm{P}, 2}^{(i)}\right) \rightarrow\left(-\bar{y}_{\mathrm{P}, 1}^{(i)}, \bar{y}_{\mathrm{P}, 2}^{(i)}\right),\left(\bar{x}_{\mathrm{P}, 1}^{(i)},-\bar{x}_{\mathrm{P}, 2}^{(i)}\right) \\
& \left(\bar{y}_{\mathrm{P}, 1}^{(i)}, \bar{y}_{\mathrm{P}, 2}^{(i)}\right),\left(\bar{x}_{\mathrm{P}, 1}^{(i)}, \bar{x}_{\mathrm{P}, 2}^{(i)}\right) \rightarrow\left(\bar{y}_{\mathrm{P}, 1}^{(i)},-\bar{y}_{\mathrm{P}, 2}^{(i)}\right),\left(-\bar{x}_{\mathrm{P}, 1}^{(i)}, \bar{x}_{\mathrm{P}, 2}^{(i)}\right) .
\end{aligned}
$$

In view of the fact that the angles $\iota_{1}, \iota_{2}$ remain unchanged under (57), we shall have that the coordinates $\left(y^{(i)}, x^{(i)}\right)=\left(\left(y_{1}^{(i)}, y_{2}^{(i)}, y_{3}^{(i)}\right),\left(x_{1}^{(i)}, x_{2}^{(i)}, x_{3}^{(i)}\right)\right)$ into the formulae (41) undergo the following transformations

$$
\begin{aligned}
& \left(y_{1}^{(i)}, y_{2}^{(i)}, y_{3}^{(i)}\right),\left(x_{1}^{(i)}, x_{2}^{(i)}, x_{3}^{(i)}\right) \rightarrow\left(-y_{1}^{(i)}, y_{2}^{(i)}, y_{3}^{(i)}\right),\left(x_{1}^{(i)},-x_{2}^{(i)},-x_{3}^{(i)}\right) \\
& \left(y_{1}^{(i)}, y_{2}^{(i)}, y_{3}^{(i)}\right),\left(x_{1}^{(i)}, x_{2}^{(i)}, x_{3}^{(i)}\right) \rightarrow\left(y_{1}^{(i)},-y_{2}^{(i)},-y_{3}^{(i)}\right),\left(-x_{1}^{(i)}, x_{2}^{(i)}, x_{3}^{(i)}\right)
\end{aligned}
$$

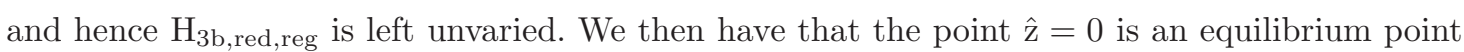
for the averaged secular perturbation, still in the case the reduction is performed completely. This equilibrium turns out to be elliptic in the case of small mutual inclination; it is hyperbolic for large inclinations. In correspondence of such situations, unstable tori with two frequencies for large inclinations have been found in [18]; stable tori with four (maximal) frequencies have been found in [27], when the inclinations are small.

The situation is rather different when $n \geq 3$. In that case, we have to face up formulae (56). Such formulae have a more complicate structure than (41). It is possible to see that (58) holds for $i=n-1, n$, but not in general. And in fact it turns out that the Taylor expansion of secular perturbing function around $\hat{z}=0$ for the fully reduced system described in $\S 4.3 .2$ contains also powers with odd degree. The point $\hat{z}=0$ is no longer an equilibrium and the construction of the Birkhoff normal form is prevented.

This problem has been treated locally in [8] interchanging the order of operations: postponing the full reduction after the Birkhoff-normalization of the partially reduced system and then applying an Implicit Function Theorem procedure definitely restores (in the range of small mutual inclinations) a suitable Birkhoff normal form (in particular, the elliptic equilibrium) also for the completely reduced system. The cost is that the reduction is defined only in the local domain of the Birkhoff transformation.

\section{5 "Perihelia reduction": A symmetric full reduction of $\mathrm{SO}(3)$ invari- ance}

In this section we present Kepler map for the planetary problem which reduces the number of degrees of freedom to $(3 n-2)$ and, simultaneously, keeps memory of reflection invariance for any $n \geq 2$.

This map

$$
\left(\phi_{\mathrm{P}_{*}}\right)^{-1}: \quad\left(y^{(1)}, \cdots, y^{(n)}, x^{(1)}, \cdots, x^{(n)}\right) \rightarrow \mathrm{P}_{*}=(\Lambda, \chi, \Theta, \ell, \kappa, \vartheta)
$$


where

$$
\begin{array}{ll}
\Lambda=\left(\Lambda_{1}, \cdots, \Lambda_{n}\right) \in \mathbb{R}^{n} & \ell=\left(\ell_{1}, \cdots, \ell_{n}\right) \in \mathbb{T}^{n} \\
\chi=\left(\chi_{0}, \bar{\chi}\right) \in \mathbb{R} \times \mathbb{R}^{n-1} & \kappa=\left(\kappa_{0}, \bar{\kappa}\right) \in \mathbb{T} \times \mathbb{T}^{n-1} \\
\Theta=\left(\Theta_{0}, \bar{\Theta}\right) \in \mathbb{R} \times \mathbb{R}^{n-1} & \vartheta=\left(\vartheta_{0}, \bar{\vartheta}\right) \in \mathbb{T} \times \mathbb{T}^{n-1}
\end{array}
$$

with

$$
\begin{aligned}
& \bar{\chi}=\left(\chi_{1}, \cdots, \chi_{n-1}\right), \quad \bar{\kappa}=\left(\kappa_{1}, \cdots, \kappa_{n-1}\right) \\
& \bar{\Theta}=\left(\Theta_{1}, \cdots, \Theta_{n-1}\right), \quad \bar{\vartheta}=\left(\vartheta_{1}, \cdots, \vartheta_{n-1}\right)
\end{aligned}
$$

is defined as follows.

Let, as in $\S 3.2, \mathfrak{m}_{1}, \cdots, \mathfrak{m}_{n}, \mathfrak{M}_{1}, \cdots, \mathfrak{M}_{n}$ be fixed mass parameters; let $a^{(i)} \in \mathbb{R}_{+}, e^{(i)}, P^{(i)} \in \mathbb{R}^{3}$, with $\left|P^{(i)}\right|=1$, denote the semi-major axis, eccentricity, the direction of the perihelion of the $i^{\text {th }}$ instantaneous ellipse $\mathfrak{E}_{i}=\mathfrak{E}_{i}\left(y^{(i)}, x^{(i)}\right)$ generated by the two-body Hamiltonian $h_{2 \mathrm{~b}}^{(i)}$ in (7) with initial datum $\left(y^{(i)}, x^{(i)}\right)$; let $\mathcal{A}^{(i)}$ the area spanned by $x^{(i)}$ on $\mathfrak{E}_{i}$ with respect to $P^{(i)}$. Let and $\mathrm{C}^{(i)}, \mathrm{S}^{(i)}$ be as in (47). Define, finally, the following $n$ couples of nodes, $\left(\widetilde{\nu}_{j}, \widetilde{\mathrm{n}}_{j}\right)_{1 \leq j \leq n}$

$$
\widetilde{\nu}_{1}:=k^{(3)} \times \mathrm{C}, \quad \widetilde{\mathrm{n}}_{j}:=\mathrm{S}^{(j)} \times P^{(j)}, \quad \widetilde{\nu}_{j+1}:=P^{(j)} \times \mathrm{S}^{(j+1)}, \quad \widetilde{\mathrm{n}}_{n}:=P^{(n)}
$$

with $1 \leq j \leq n-1$. Assume that such nodes do not vanish. Then let

$$
\begin{aligned}
& \Theta_{j-1}=\left\{\begin{array}{lr}
\mathrm{C}_{3}:=\mathrm{C} \cdot k^{(3)} \\
\mathrm{S}^{(j)} \cdot P^{(j-1)}
\end{array} \quad \vartheta_{j-1}=\left\{\begin{array}{lr}
\zeta:=\alpha_{k^{(3)}}\left(k^{(1)}, \widetilde{\nu}_{1}\right) & j=1 \\
\alpha_{P^{(j-1)}}\left(\widetilde{\mathrm{n}}_{j-1}, \widetilde{\nu}_{j}\right) & 2 \leq j \leq n
\end{array}\right.\right. \\
& \chi_{j-1}:=\left\{\begin{array}{lr}
\mathrm{G}=\left|\mathrm{S}^{(1)}\right| \\
\left|\mathrm{S}^{(j)}\right|
\end{array} \quad \kappa_{j-1}:=\left\{\begin{array}{lr}
\mathfrak{g}:=\alpha_{\mathrm{S}^{(1)}}\left(\widetilde{\nu}_{1}, \widetilde{\mathrm{n}}_{1}\right) & j=1 \\
\alpha_{\mathrm{S}^{(j)}}\left(\widetilde{\nu}_{j}, \widetilde{\mathrm{n}}_{j}\right) & 2 \leq j \leq n
\end{array}\right.\right. \\
& \Lambda_{i}:=\mathfrak{M}_{i} \sqrt{\mathfrak{m}_{i} a^{(i)}} \quad \quad \ell_{i}:=2 \pi \frac{\mathcal{A}^{(i)}}{\mathcal{A}_{\text {tot }}^{(i)}}:=\text { mean anomaly of } x^{(i)} \text { on } \mathfrak{E}_{i}
\end{aligned}
$$

The map (59) is Kepler map in the sense of $\$ 3.2$. Relations (24)-(25) hold by definition of the instantaneous ellipses $\mathfrak{E}_{i}\left(y^{(i)}, x^{(i)}\right)$.

Main points are

- The reduction realized by the variables (59) is based on a new chain of frames

$$
\mathrm{F}_{0} \rightarrow \widetilde{\mathrm{F}}_{1} \rightarrow \mathrm{G}_{1} \rightarrow \cdots \rightarrow \widetilde{\mathrm{F}}_{n} \rightarrow \mathrm{G}_{n}
$$

This chain contains $(2 n)$ changes. This may be compared with the chains (49), which instead may contain up to $n$ changes. In the chain $(62), \mathrm{F}_{0}$ is a prefixed initial frame; $\widetilde{\mathrm{F}}_{j}$, with $1 \leq j \leq n$ are frames which (analogously to the frames $\mathrm{F}_{j}$ in (49)) have their respective third axes directed towards $\mathrm{S}^{(j)}$. The frames $\mathrm{G}_{j}$ are completely new (and this is the main difference with Deprit's reduction): they have their third axes are directed towards the perihelia $P^{(j)}$ of instantaneous ellipses. For this reason we call this reduction as "Perihelia reduction", while Deprit's reduction might be named "inclinations reduction". Clearly, also the inclinations play a rôle in (62), by means of the $\widetilde{\mathrm{F}}_{j}$ 's, but the main point is that the orbital frames, with their third axes in the direction of $\mathrm{C}^{(j)}$ (corresponding to the $\mathrm{F}_{j}$ in (49)), are not part of (62). In other words, $\mathrm{C}^{(j)}$ are not independent vectors in the reduction (while $P^{(j)}$ are so) and their lengths $\Gamma_{j}=\left|\mathrm{C}^{(j)}\right|$ are not independent actions. 
- The coordinates $(\Lambda, \bar{\chi}, \bar{\Theta}, \ell, \bar{\kappa}, \bar{\vartheta}) \in \mathbb{R}^{n} \times \mathbb{R}^{n-1} \times \mathbb{R}^{n-1} \times \mathbb{T}^{n} \times \mathbb{T}^{n-1} \times \mathbb{T}^{n-1}$ are canonical coordinates for $(6 n-4)$-dimensional manifold

$$
\mathcal{M}_{\tilde{\nu}_{1}}=\left\{k^{(1)}=\tilde{\nu}_{1} \quad k^{(3)}=\mathrm{C}\right\} .
$$

Such manifold corresponds to fix a rotating frame with the third axis parallel to $\mathrm{C}$ and such that, with respect to it, the node $\tilde{\nu}_{1}$ determined by the plane orthogonal to the first perihelion $P^{(1)}$ is fixed. This should be compared with the corresponding manifold (34) of Deprit's reduction.

- Singularities of the coordinates (61) appear in correspondence of vanishing eccentricities (in which case the perihelia $P^{(i)}$ are not defined) or cases of parallelism of two consecutive frames in the chain (62). The parallelism between two frames $\widetilde{\mathrm{F}}_{j}, \widetilde{\mathrm{F}}_{j+1}$ is not a singularity. In particular, the coordinates (61) are well defined also in the case of planar limit (which corresponds to all the $\widetilde{\mathrm{F}}_{j}$ 's parallel one with another); contrarily to what happens for JacobiRadau and Boigey-Deprit's reductions.

- The fact that the perihelia $P^{(i)}$ are independent directions allows for a symmetry by reflections. (In the reductions by Boigey-Deprit, the directions of the perihelia are not independent, since they are constrained to be orthogonal to the $\mathrm{C}^{(j)}$ 's, which are independent directions.) Transformations

$$
(\Lambda, \chi, \Theta, \ell, \kappa, \vartheta) \rightarrow(\Lambda, \chi,-\Theta . \ell, \kappa, 2 k \pi-\vartheta) \quad k \in \mathbb{Z}^{n}
$$

correspond to

$$
\left(\left(y_{1}^{(i)}, y_{2}^{(i)}, y_{3}^{(i)}\right),\left(x_{1}^{(i)}, x_{2}^{(i)}, x_{3}^{(i)}\right)\right) \rightarrow\left(\left(y_{1}^{(i)},-y_{2}^{(i)}, y_{3}^{(i)}\right),\left(x_{1}^{(i)},-x_{2}^{(i)}, x_{3}^{(i)}\right)\right) .
$$

The coordinates (61) have been presented in the note [25], together with a sketchy illustration of an application of them. The proof of their canonical character (and of the result announced in that note) is deferred to a subsequent paper.

\section{References}

[1] Khaled Abdullah and Alain Albouy: On a strange resonance noticed by M. Herman, Regul. Chaotic Dyn., 6, 4, 421-432 (2001)

[2] Vladimir I. Arnol'd: Small denominators and problems of stability of motion in classical and celestial mechanics, Uspehi Mat. Nauk, 18(6 (114)):91-192, (1963). English translation: Russian Math. Surv. 18, 6, 85-191 (1963).

[3] Françoise Boigey: Élimination des nœuds dans le problème newtonien des quatre corps, Celestial Mech., 27, 4, 399-414 (1982)

[4] Luigi Chierchia: The Planetary N-Body Problem, UNESCO Encyclopedia of Life Support Systems, 6.119.55, (2012)

[5] Luigi Chierchia and Gabriella Pinzari: Properly-degenerate KAM theory (following V. I. Arnold), Discrete Contin. Dyn. Syst. Ser. S, 3(4):545-578 (2010)

[6] Luigi Chierchia and Gabriella Pinzari: Deprit's reduction of the nodes revisited, Celestial Mech. Dynam. Astronom., 109, 3, 285-301 (2011)

[7] Luigi Chierchia and Gabriella Pinzari: Planetary Birkhoff normal forms, J. Mod. Dyn., $5(4): 623-664(2011)$ 
[8] Luigi Chierchia and Gabriella Pinzari: The planetary $N$-body problem: symplectic foliation, reductions and invariant tori, Invent. Math., 186, 1, 1-77 (2011)

[9] Luigi Chierchia and Gabriella Pinzari: Metric stability of the planetary $N$-body problem, Proceedings of the Iinternational Congress of Mathematicians (2014)

[10] André Deprit: Elimination of the nodes in problems of $n$ bodies, Celestial Mech., 30(2):181195 (1983).

[11] Jacques Féjoz: Démonstration du 'théorème d'Arnold' sur la stabilité du système planétaire (d'après Herman), Ergodic Theory Dynam. Systems, 24(5):1521-1582 (2004).

[12] Jacques Féjoz: On "Arnold's theorem" on the stability of the solar system, Discrete Contin. Dyn. Syst., 33(8):3555-3565 (2013).

[13] Jacques Féjoz, Marcel Guardia, Vadim Kaloshin, and Pablo Roldan: Diffusion along mean motion resonances in the restricted planar three-body problem, J. Eur. Math. Soc. (2014) To appear.

[14] Sebastián Ferrer and Carlos Osácar: Harrington's Hamiltonian in the stellar problem of three bodies: reductions, relative equilibria and bifurcations, Celestial Mech. Dynam. Astronom., $58,3,245-275$ (1994)

[15] Robert S. Harrington: The stellar three-body problem, Celestial Mechanics, 1, 2, 200-209 (1969)

[16] Michael Robert Herman: Torsion du problème planétaire, http://www.college-defrance.fr/default/EN/all/equ_dif/archives_michel_herman.htm (2009)

[17] Par M. Jacobi: Sur l'élimination des noeuds dans le probléme des trois corps, Astronomische Nachrichten, 20, 6, 81-98 (1843)

[18] William H. Jefferys and Jürgen Moser: Quasi-periodic solutions for the three-body problem, Astronom. J., 71, 568-578 (1966)

[19] Jacques Laskar and Philippe Robutel: Stability of the planetary three-body problem. I, Expansion of the planetary Hamiltonian,Celestial Mech. Dynam. Astronom., 62, 3, 193-217 (1995).

[20] Michail L. Lidov and S. L. Ziglin: Non-restricted double-averaged three body problem in Hill's case, Celestial Mech., 13(4):471-489 (1976)

[21] Frank Malige, Philippe Robutel, and Jacques Laskar: Partial reduction in the $n$-body planetary problem using the angular momentum integral, Celestial Mech. Dynam. Astronom., 84, $3,283-316(2002)$

[22] Nikholai N. Nekhoroshev: An exponential estimate of the time of stability of nearly integrable Hamiltonian systems, Uspehi Mat. Nauk, 32(6(198)):5-66, 287 (1977).

[23] Gabriella Pinzari: On the Kolmogorov set for many-body problems, PhD thesis, Università Roma Tre, arXiv:1309.7028 (2009)

[24] Gabriella Pinzari: Aspects of the planetary Birkhoff normal form, Regul. Chaotic Dyn., 18, 6, 860-906 (2013)

[25] Gabriella Pinzari: Global Kolmogorov tori in the planetary $N$-body problem. Announcement of result, arXiv:1406.4493 (2014) 
[26] J. C. Rodolphe Radau: Sur une transformation des équations différentielles de la dynamique, Ann. Sci. École Norm. Sup., 5:311-375 (1868).

[27] Philippe Robutel: Stability of the planetary three-body problem. II. KAM theory and existence of quasiperiodic motions, Celestial Mech. Dynam. Astronom., 62, 3:219-261 (1995)

[28] Edmund Taylor Whittaker: A treatise on the analytical dynamics of particles and rigid bodies: With an introduction to the problem of three bodies, 4th ed. Cambridge University Press, New York (1959)

[29] Lei Zhao: Quasi-periodic solutions of the spatial lunar three-body problem, Celestial Mech. Dynam. Astronom., 119, 1, 91-118 (2014) 